\title{
We can guide search by a set of colors, but are reluctant to do it
}

\author{
Michael J. Stroud ${ }^{1} \cdot$ Tamaryn Menneer $^{2} \cdot$ Elina Kaplan $^{3} \cdot$ Kyle R. Cave $^{4} \cdot$ Nick Donnelly $^{5}$ \\ Published online: 6 November 2018 \\ (C) The Psychonomic Society, Inc. 2018
}

\begin{abstract}
For some real-world color searches, the target colors are not precisely known, and any item within a range of color values should be attended. Thus, a target representation that captures multiple similar colors would be advantageous. If such a multicolor search is possible, then search for two targets (e.g., Stroud, Menneer, Cave, and Donnelly, Journal of Experimental Psychology: Human Perception and Performance, 38(1): 113-122, 2012) might be guided by a target representation that included the target colors as well as the continuum of colors that fall between the targets within a contiguous region in color space. Results from Stroud, Menneer, Cave, and Donnelly, Journal of Experimental Psychology: Human Perception and Performance, 38(1): 113-122, (2012) suggest otherwise, however. The current set of experiments show that guidance for a set of colors that are all from a single region of color space can be reasonably effective if targets are depicted as specific discrete colors. Specifically, Experiments 1-3 demonstrate that a search can be guided by four and even eight colors given the appropriate conditions. However, Experiment 5 gives evidence that guidance is sometimes sensitive to how informative the target preview is to search. Experiments 6 and 7 show that a stimulus showing a continuous range of target colors is not translated into a search target representation. Thus, search can be guided by multiple discrete colors that are from a single region in color space, but this approach was not adopted in a search for two targets with intervening distractor colors.
\end{abstract}

Keywords Color search $\cdot$ Dual-target cost $\cdot$ Eye movements $\cdot$ Top-down search guidance $\cdot$ Search representation $\cdot$ Split-target cost

\section{Introduction}

Visual search tasks often involve a single known target that must be located within a field of distractors. Search efficiency is dictated by the internal representation of the search target (top-down) and the relationship of that target to the distractor objects to be searched through (bottom-up). In terms of topdown guidance, typically observers are given the exact object

Michael J. Stroud

stroudm@merrimack.edu

1 Department of Psychology, Merrimack College, O’Reilly Hall, 315 Turnpike Street, North Andover, MA 01845, USA

2 The European Centre for Environment and Human Health, University of Exeter Medical School, Royal Cornwall Hospital, Truro, UK

3 Neuroscience and Behavior Program, University of Massachusetts, Amherst, MA, USA

4 Department of Psychological and Brain Sciences, University of Massachusetts, Amherst, MA, USA

5 Department of Psychology, Liverpool Hope University, Liverpool, UK to search for, and that item is held in some form of memory during search, which might be a type of working memory (Desimone \& Duncan, 1995; Olivers, Peters, Houtkamp, \& Roelfsema, 2011) or a long-term representation (Cunningham \& Wolfe, 2014; Wolfe, 2012; Wolfe, Aizenman, Boettcher, \& Cain, 2016). Representation of a single target (e.g., a red horizontal bar) activates the relevant features to guide search, and objects that share features with the search target receive attention (red objects and horizontally-oriented objects). These are often referred to as the attentional control settings or mechanisms of the observer (Folk, Remington, \& Johnston, 1992). These allow the searcher to narrow attention to the relevant features determined by the search task.

Color is a feature that is often used to find targets very effectively (Treisman \& Gelade, 1980; Wolfe, Cave \& Franzel, 1989). Wolfe \& Horowitz (2004) suggested that color contains "undoubted attributes" that guide attention. For the current research, color is useful because it readily attracts eye movements, which allow one to infer characteristics of the internal mental representation that is guiding search (Williams, 1967; Williams \& Reingold, 2001).

Although the majority of laboratory search tasks involve a single target, some tasks include more than one target, and 
sometimes there is limited information known about the target. A common practical example of this type of dual-target search arises when someone is trying to locate specific items on a crowded desk. The observer may be trying to locate a silver pen-shaped laser pointer as well as a blue pen before leaving for a lecture. In this example, both objects share a common feature (shape), but differ in another feature dimension (color). Can the observer efficiently search for both objects simultaneously?

There is some evidence of efficient search for multiple targets that share the same color, but differ by shape (Menneer, Cave \& Donnelly, 2009; Stroud, Menneer, Cave, Donnelly, \& Rayner, 2011), for highly similar colored targets (Stroud, Menneer, Cave, \& Donnelly, 2012), and for semantically related targets (Sobel, Puri, \& Hogan, 2015). However, there is a clear cost in accuracy and/or time in search for multiple targets among real-world objects (Hout \& Goldinger, 2010; Menneer et al., 2009; Menneer, Stroud, Cave, Li, Godwin, Liversedge, \& Donnelly, 2012), abstract representations of real-world objects (Menneer, Donnelly, Godwin \& Cave, 2010), and color patches, orientations and abstract shapes, (Menneer, Barrett, Phillips, Donnelly \& Cave, 2004, 2007).

Even though some of these studies show a large drop in performance between one-target search and two-target search, subjects are nonetheless able under the right circumstances to search reasonably effectively for a large set of possible targets, as demonstrated by Wolfe, Aizenman, Boettcher, and Cain (2016), who tested searches for up to 64 different targets. Wolfe (2012) used an even larger target set (up to 100 objects) and showed a cost in search response time, which increased with the $\log$ of the target set size. It is unclear whether the mechanisms that produce this response time (RT) cost with large target sets are also responsible for the costs found with smaller target sets. For instance, as discussed later, large-targetset search may differ from small-target-set search in how target representations are distributed between working memory and long-term memory. The current set of experiments will focus on the costs arising from searching for relatively small numbers of target values within the color dimension.

Stroud et al. (2012) investigated the dynamics of multi-target search for color by observing eye movements during search for one versus two color targets. Three different sets of two-color target-pairs were used, each having a different level of target similarity within color space. The fixation data in all conditions showed that search was guided with varying degrees of accuracy by the target color. The results revealed two sources of costs associated with maintaining multiple representations during search. First, there is a clear cost for searching for two colors over separate individual searches (dual-target cost). The second type of cost appears to be related to the similarity between the two search targets. As the similarity between the targets decreases, the color selectivity decreases, i.e., the number of fixations directed to non-target colors increases. For instance, if the observer is searching for a red and blue object, then a large proportion of fixations are directed to yellow and green objects. Stroud et al., termed this a "split-target" cost, and it represents the decrement in search guidance associated with a split or separation of target values in color space. Both the dual-target and split-target costs appear to contribute to the decrease in color selectivity compared to single-target searches.

There are several possible ways that multiple targets can be mentally represented to guide search. First, there is the possibility that separate representations or templates are constructed for each search target. Second, multiple items may be represented by a unified set of possible target values or a single, undifferentiated template that extends over the target-color region in color space (e.g., Hout \& Goldinger, 2015; Stroud et al., 2012). The first option requires the ability to maintain multiple separate representations to guide search, while the second requires the ability to construct a single representation that covers a set of similar colors.

In terms of target representations held in memory, the majority of evidence supports the first account described above, that two separate representations can be maintained (Barrett \& Zobay, 2014; Beck, Hollingworth \& Luck, 2012; Irons, Folk \& Remington, 2012; Stroud et al., 2012; Moore \& Weissman, 2010). Stroud et al. (2012) applied a discrete target combination model to show that both similarly colored targets (e.g., orange and yellow) and dissimilarly colored targets (e.g., orange and green) could be represented by two discrete representations. The model demonstrated that the fixation rates for distractor colors that fall in-between the targets in color space were explained by the combination of fixation rates to colors that are outside the range of the targets.

The two separate target representations may guide search simultaneously, or control might alternate between them. Results from Cave, Menneer, Nomani, Stroud, and Donnelly (2018) seem to show control alternating between the two targets, but evidence from Beck et al. (2012; see also Hollingworth \& Beck, 2016; Beck \& Hollingworth, 2017) suggests that two targets can be maintained and guide search simultaneously. They found a switch cost (in time) when subjects searched for one target followed by the other, but no switch cost when instructed to search for both targets simultaneously. The lack of the cost suggests both target representations were maintained and active during the search. In search for two colors, Irons et al. (2012) found that irrelevant cues that matched either of the target colors resulted in a spatial cueing effect. In other words, both target colors were capturing attention. They concluded that it may be possible to maintain two simultaneous color templates. Performance seemed to be lower with two targets than with one, suggesting that search guidance was slowed due to the working memory load and resource competition from the extra target representation.

Barrett and Zobay (2014) compared single-representation and dual-representation models against dual-target search performance, and found the best fit with a dual-representation 
model comprising simultaneous noisy representations. They concluded that limited capacity and the requirement to maintain two representations results in reduced resources for maintaining the specificity of information held in memory, thereby impacting search guidance and object identification.

In contrast, Houtkamp and Roelfsema (2009) provided evidence against two separate representations in working memory being used simultaneously to identify a target. Rather than searching in a spatial array, their subjects searched for two targets in a stream of stimuli presented one after another at the same location. There was a cost in accuracy in searching for two targets compared to one, and that cost was captured by their single-template model, while performance was overestimated by the dual-template model. These results can be compared with those of Moore \& Weismann $(2011,2010$, 2014), who found that initially subjects were able to search for two target colors as well as one, but that after a distractor appeared with one of the target colors, there were more errors at detecting the other target color. However, given that stimuli were presented one at a time in all of these studies, these findings apply to the process of matching the current item to the target representation, and not to the search guidance process. As with the other studies, resource limitations were advocated as the cause of the cost.

The growing body of research surrounding representation of multiple targets demonstrates flexibility in attentional guidance, but that flexibility has costs. Generally speaking, it appears the costs are based on the difficulty in maintaining separate representations or templates, resulting in a competition for attentional resources or working memory space. It is not surprising that storing two target representations to guide search would use more resources than storing one target, especially if they are being stored in a working memory that is organized into a small number of slots. However, it would be surprising if the attentional system had no efficient way of searching for a target color that might fall anywhere within a well-defined region within color space. In many real-world searches, the exact color of the target cannot be known in advance because of variations in illumination and in the reflectance properties of targets. For instance, the apples on a tree will not all be exactly the same shade of red, and some parts of the tree may be more shaded than others. It would be very useful to be able to represent the target color not just as a point within color space, but as a region. If that unified region or range representation occupied just one slot in working memory, it could lead to efficient search. And even if the memory representation guiding search is organized as a flexible resource rather than as slots, a unified range representation might still require fewer resources than multiple separate target representations.

There is evidence for the use of a single template for similar targets (Hout \& Goldinger, 2015), so it may be possible to guide search for multiple colors by a single unified target template. However, the results from Stroud et al. (2012) show little evidence for this type of guidance, and instead indicate guidance targeted specifically at the two possible target colors. That study compared three separate dual-target color search conditions against search for a single target color. In the dualtarget conditions, the relative similarity between the two target colors was varied, which directly affected how the fixations were distributed across items with higher and lower similarity to the target colors. The conditions that are relevant to the current study are those in which distractor colors appeared between the two targets in color space. In the similar (twostep) condition, the target colors were two steps away in the ring of 16 possible colors that defined the color space used in these experiments (see Fig. 1 later), with one intervening distractor color. For the dissimilar (four-step) condition, the targets were spaced four steps apart on the ring, leaving three possible distractor colors in between. In the two-step condition, the intermediate distractor color received the same level of fixations as the two targets, while the three intervening distractors in the four-step condition received considerably fewer fixations compared to the targets. The discrete target combination model suggested that the patterns in both conditions were consistent with two separate representations. However, and importantly for the current study, the individual subject data suggest that some subjects represented the two targets as a range, fixating the intervening colors at the same rate as the target colors, even with four steps between targets. Other subjects attempted to hold two separate object representations, producing fewer fixations to intervening colors than target colors.

Some aspects of Stroud et al.'s (2012) search task probably discouraged the use of a single unified template to guide search. The current study will explore this issue in two steps.

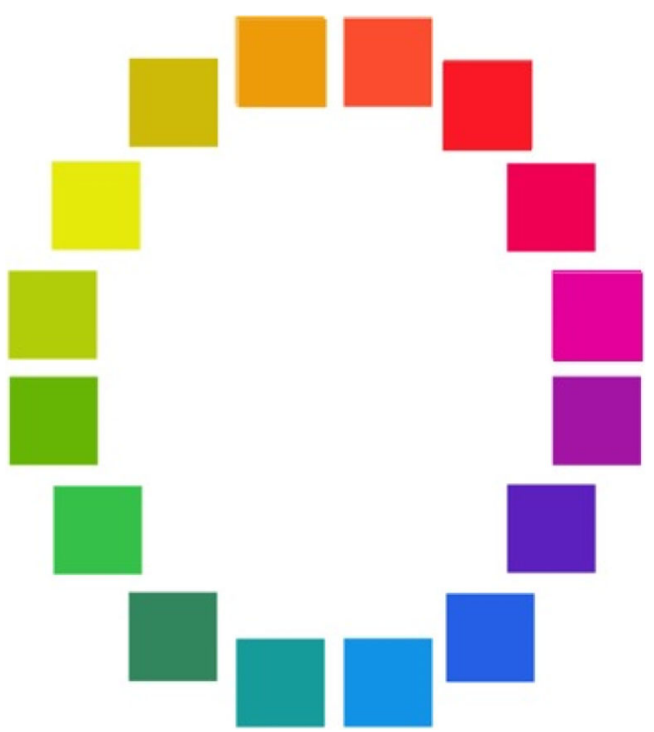

Fig. 1 The ring of 16 colors assigned to the search stimuli across all experiments 
The first four experiments will test whether it is possible to guide search by a large set of colors that are adjacent to each other on the ring of colors (see Fig. 1) used in these experiments, and therefore a contiguous region in color space contains only target colors and not distractor colors. To encourage the adoption of a representation that encompasses all of the target colors, key aspects of the stimuli and procedure are changed from Stroud et al. As in Stroud et al., the pattern of fixations to different colors is used to reveal the search process, and to allow us to draw conclusions about the nature of the search representation. To preview the results: there is a fairly strong level of color guidance for large sets of colors that come from a contiguous distractor-free region in color space. Guidance is stronger when the target colors are similar to one another.

The evidence from Experiments 1-3 that search can be guided by a large set of similar colors makes it more plausible that search can be guided by a unified template that represents a range of colors. Therefore, Experiments 5-7 in the current study will explore whether conditions can be changed in Stroud et al. (2012)'s original dual-target search task to encourage the use of such a range representation, despite target colors spanning a section of color space that includes distractor colors. As described below, the results show that despite changes to the presentation of the target colors to the subjects in Experiments 5 and 6, these experiments produced no evidence for an effective range representation of the target colors in the Stroud et al. search task.

Another factor is manipulated in Experiment 7, which tests whether a unified target representation can be encouraged in dual-target search by removing colors that fall between the two target colors from the search display. However, the manipulation had no effect on search guidance.

In summary, the current study addresses two main questions. The first is whether search can be guided by a set of target colors that can be captured by a representation comprising a single contiguous region of color space that does not include distractor colors. If search can be guided by such a set of colors, the second question is whether such guidance can be promoted in dual-target search either by presenting target colors as part of a continuum, or by removing distractor colors that fall between the targets.

\section{Experiment 1}

Experiment 1 was similar to Stroud et al.'s (2012) experiment in that subjects determined whether a target was present or absent in a search array. Both color and shape information were relevant for finding targets. Some items that had one of the target colors were actually distractors, and only targets had a $\mathrm{T}$ shape. The presence of these target-color distractors makes it possible to determine how much of the guidance is based on color and not shape.

One factor that may have encouraged subjects to maintain separate templates for the two target colors in some conditions of Stroud et al. (2012) is that the two target colors were separated in color space by other colors that were never targets. This arrangement may make it more difficult to represent the target colors as a single unified set. Also, when deciding how to organize a template to guide search, subjects might use the information that they accumulate over the course of the experiment about the relative proportions of the targets and distractors. In Stroud et al. (2012), the distractors with a target color appeared frequently enough that when an item appeared with the target color, it was more likely to be a distractor than a target. Observers may therefore be less likely to use the target color(s) to guide attention, simply because those colors appear more frequently as distractors.

To encourage a target representation comprising a set of contiguous colors, the current experiment differed in three ways from Stroud et al.: (1) the target preview consisted of four discrete color patches to search for, (2) the targets could appear in any of the four colors (compared to just the two used previously), and (3) when an object was the color of the target, it was more likely to be a target rather than a distractor. In Stroud et al.'s original experiment, only $30 \%$ of the objects with a target color were actual targets. In the high-targetprobability (HTP) condition of the current experiment, $62 \%$ of the target-color items were targets. With these changes, the preview of the possible targets shown at the beginning of each trial was more informative than the preview used in Stroud et al., because the information provided had a higher chance of leading to a target. It is important to note that in the current experiment, the target could have any of four different colors, which may reach the limits of visual working memory capacity of three to four items (Luck \& Vogel, 1997). Thus, there is the possibility that the system will be forced to lower the working memory load by creating a unified range of colors or by chunking the colors together to guide search.

\section{Method}

\section{Subjects}

Sixteen subjects recruited from the University of Massachusetts community took part in Experiment 1 . The subjects had a mean age of 20.6 years $(S D=1.7)$.

\section{Design}

The main data analyses were based on fixation rates of the different colored distractors that appeared in the search arrays. The design consisted of three factors. Color Step represented the distractor's color relative to whichever of the target colors was 
most similar to it. In order to quantify this difference, Color Step was set to the number of steps between the distractor color and the closest target color around the ring of 16 stimulus colors (Fig. 1). With four possible target colors, there are 12 distractor colors that never appear as targets, with two of these colors falling at each distance (one to six steps) from their most similar target. In this analysis, the factor of color step includes those six levels for the non-target colors, along with one level for the two target colors at the outermost edge of the target region in color space, for a total of seven levels. The colors that fell between the two outermost target colors were not included in this analysis. The second factor was Target Set, which is the set of colors that could be assigned to a target item. This factor had two levels: (1) fourstep, which had two target colors that were four steps apart on the color ring and low probability that a target-color item would be a target and (2) four-color HTP, with four target colors from adjacent steps on the color ring and high target probability (HTP). The data for the first level were taken from the four-step condition from Stroud et al. (2012) for comparison. The third factor was Target Presence (absent vs. present). The resulting design was a 2 (Target Set: four-step vs. four-color HTP) $\times 2$ (Target Presence: Absent vs. Present) $\times 7$ (Color Step) mixed factorial design with Target Set as the only between-subjects factor.

The primary dependent measure was the probability that an object with a particular color would be fixated once or more during each trial in which it appeared. Secondary dependent measures included RT and error rates.

\section{Apparatus and stimuli}

Each subject's right eye was tracked as they viewed the stimuli on a 17-in. Vision Master Pro 514 iiyama CRT monitor attached to a computer interfaced with an SR Research Limited Eye-Link II eye tracking system operating at a sampling rate of $250 \mathrm{~Hz}$. Stimuli were presented $57 \mathrm{~cm}$ from the subject with the entire display subtending $25.7^{\circ} \times 32.5^{\circ}$ of visual angle. Subjects viewed the stimuli with binocular vision while their head was kept stationary in a chin rest. Corneal reflections and pupil position were tracked and the equipment was recalibrated if there was ever greater than $0.40^{\circ}$ of visual angle error at calibration.

In both conditions, the search objects consisted of " $\mathrm{T}$ "s (targets) and "L"s (distractors) that were constructed from two rectangles measuring $1.04^{\circ} \times 0.37^{\circ}$ of visual angle. One rectangle was joined at the midline of the broad side of another rectangle to form the $\mathrm{T}$, resulting in $0.5^{\circ}$ of offset. In order to reduce $\mathrm{T} / \mathrm{L}$ discriminability and encourage more fixations, the pseudo Ls had the rectangles joined with an offset of $0.3^{\circ}$ on the short side and $0.7^{\circ}$ on the long side.

Sixteen possible colors were used for the objects throughout the experiment. The pool of colors was spaced in a ring in CIExyY color space and was selected to maximize the use of the available color gamut of the monitor in order to have differences beyond the just-noticeable differences (Wyszecki and Stiles, 1982). Achieving visually distinct colors required using different luminances across the colors given that 16 isoluminant hues would be much more difficult to distinguish. Hues and spacing were also chosen to minimize any single color visibly "popping out" from the rest (see Fig. 2). We used the same collection of colors as in Menneer et al. (2007, 2010) and Stroud et al. $(2010,2012)$ to allow comparison with the previous experiments of Stroud et al. (2012). Please see Table 1 of Stroud et al. for the CIExyY coordinates of the colors. Luminance (Y) values ranged from 0.11 to 0.78 as a proportion of white luminance. While the variance in luminance across stimulus colors might be considered a limitation in control, any effects due to differences across the specific colors were avoided by using all colors as targets, balanced across subjects, as in previous studies. Some discussion of the variation across target colors is provided in the Results and Discussion sections of Experiments 3 and 4, in the context of interpreting the findings of Experiments 1-4.

In each search array, ten objects were equally spaced around an imaginary circle with a radius of $9.8^{\circ}$ of visual angle for each stimulus display. Each object was an L, with the exception of target-present trials in which a single $\mathrm{T}$ was the target. The objects were presented at one of four orientations determined randomly from $0^{\circ}, 90^{\circ}, 180^{\circ}$, or $270^{\circ}$ (see Fig. 2 for a sample array).

A different set of four target colors was used for each subject, such that all colors were used as targets equally often across the experiment. The four target colors for a given subject were adjacent on the color ring (Fig. 1). Each target appeared with one of the four target colors specified for that subject. Each distractor was drawn from a pool of objects without replacement, so that some search arrays had more color variation than others. The frequencies of distractor and target colors were designed to encourage the use of color to guide search. In both conditions, there are 128

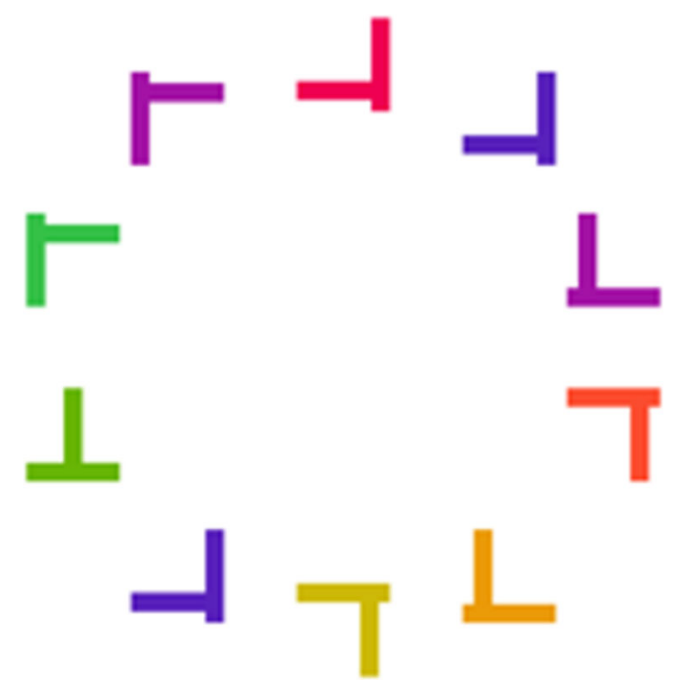

Fig. 2 An example of the search array for a target-present trial with a target $\mathrm{T}$ and distractor Ls 
Table 1 Summary of the effect size for Color Step and the main conclusion for each experiment

\begin{tabular}{|c|c|c|c|c|c|}
\hline Experiment & Main manipulation & $\begin{array}{l}\text { Number of } \\
\text { target } \\
\text { colors }\end{array}$ & $\begin{array}{l}\text { Distance between } \\
\text { least similar target } \\
\text { colors }\end{array}$ & $\begin{array}{l}\text { Effect size for Color } \\
\text { Step (i.e., guidance } \\
\text { strength) }\end{array}$ & Main conclusion \\
\hline $\begin{array}{l}\text { Stroud et al. } \\
\quad(2012)\end{array}$ & Four-step & 2 & 4 & .820 & $\begin{array}{l}\text { For comparison with current effect } \\
\text { sizes }\end{array}$ \\
\hline $\begin{array}{l}\text { Stroud et al. } \\
\quad(2012)\end{array}$ & Two-step & 2 & 2 & .859 & $\begin{array}{l}\text { For comparison with current effect } \\
\text { sizes }\end{array}$ \\
\hline 1 & $\begin{array}{l}\text { Four-color HTP (High target probability: } \\
\text { Target colors have a high probability of } \\
\text { being the target) }\end{array}$ & 4 & 3 & .780 & $\begin{array}{l}\text { No significant difference in } \\
\text { guidance strength from Stroud } \\
\text { et al. four-step }\end{array}$ \\
\hline 2 & $\begin{array}{l}\text { Four-color LTP } \\
\text { (Low target probability) }\end{array}$ & 4 & 3 & .723 & $\begin{array}{l}\text { With four target colors, no } \\
\text { significant effect of target } \\
\text { probability }\end{array}$ \\
\hline 3 & Eight-color HTP & 8 & 7 & .693 & $\begin{array}{l}\text { With eight target colors, search } \\
\text { guidance is possible }\end{array}$ \\
\hline 4 & Eight-color LTP & 8 & 7 & .099 & $\begin{array}{l}\text { Guidance is eliminated when the } \\
\text { target probability is low }\end{array}$ \\
\hline 5 & Four-step range & 2 & 4 & .425 & $\begin{array}{l}\text { Severely reduced guidance } \\
\text { compared with four-step }\end{array}$ \\
\hline 6 & Two-step range & 2 & 2 & .738 & $\begin{array}{l}\text { Reduced guidance compared with } \\
\text { two-step, but stronger than } \\
\text { four-step range }\end{array}$ \\
\hline 7 & No-intervening, four-step & 2 & 4 & .759 & $\begin{array}{l}\text { Presence of intervening distractors } \\
\text { had no significant effect on } \\
\text { guidance }\end{array}$ \\
\hline
\end{tabular}

target-absent trials, each with ten distractors, and 128 target present trials, each with nine distractors, for a total of 2,432 distractors across all trials. In the four-step condition (from Stroud et al., 2012), distractors (non-target-color and target-color) were presented 38 times each $(\times 16$ colors $\times 4$ orientations $=2,432$ distractor items in the pool) across all the trials for a given subject. In the current experiment, the four-color HTP (high target probability) condition, non-target-color distractors were each presented 49 times and target-color distractors were each presented five times. Given that the number of target colors was doubled in the fourcolor HTP condition compared with the four-step condition, targets of each color were presented eight times ( $\times 4$ orientations) for four-color HTP across the 256 trials, as opposed to 16 in fourstep. Therefore, target-colored items were targets $62 \%$ of the time in four-color HTP compared with $30 \%$ in four-step.

Subjects in the four-color HTP condition were presented with a preview consisting of four color patches to search for, as shown in Fig. 3. Each of the search targets for a given subject appeared with one of the same four colors, i.e., the target colors for that subject remained constant throughout the entire experiment. The four colors comprised four adjacent steps on the stimulus color ring (Fig. 1) and each of the 16 possible targetcolor quadruples was assigned to one subject. Subjects
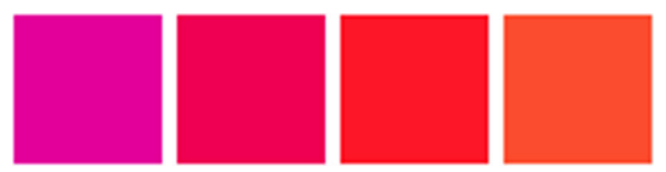

Fig. 3 An example of the target preview stimulus for Experiment 1 completed 256 experimental trials with half containing a target, preceded by five practice trials. Of the set of 128 target present trials, each of the four colored targets appeared in 32 trials.

\section{Procedure}

Prior to the experiment, subjects were administered the Ishihara test to check for any anomalies in color vision (Ishihara, 1917). Subjects were then presented with a sample display and were told they should determine whether or not a Twas present in each display. Subjects were informed that the $T$ could be any of the target colors, that one would be present on half of the trials, and that only a maximum of one T could be present in a display (a disjunctive search task). The T could have any of the four possible orientations. Responses were made on a Microsoft game controller, with the rightmost button representing "present" and the button immediately to the left signifying "absent." Subjects were told to rest their fingers on the buttons before each trial to ensure fast responses. The order of presentation for each trial consisted of (1) a central fixation point (dot) for drift correction, (2) a preview of the targets for 1,000 ms, (3) a central fixation point for $1,000 \mathrm{~ms}$, and (4) the ten-object search array until a response was made.

\section{Results}

Fixation data and response times were taken only from trials on which a correct response was given. 
Error rates and response times were submitted to a 2 (Target Set) $\times 2$ (Target Presence) ANOVA to compare performance between the four-step Target Set and four-color HTP conditions. Subjects were more accurate when the target was absent than present, $F(1,30)=96, p<.001$. When the target was present, subjects were more accurate in the four-color HTP (96.3\%) than the four-step (89.4\%), resulting in a significant Target Set $\times$ Target Presence interaction, $F(1,30)=21.43, p<.001$. In terms of response times, there was no significant difference between the two Target Sets $(p=.847)$, and the Target Set $\times$ Target Presence interaction failed to reach significance $(p=.172)$. Response time and error rate data are shown in Fig. 4.

The probability of fixating a given color was calculated as the number of objects of that color that were fixated as a proportion of the number of presentations of that object across the whole experiment. All fixations from the appearance of the search array until the response were included in the analysis. In order to determine the influence of color on search independently of shape, the fixation rates from targets (Ts) were excluded from this analysis, although fixation rates from target-colored distractors (Ls) were included.

In Stroud et al.'s four-step condition, the entire range of two targets and three intervening colors included five different colors. In the four-color HTP condition, the target range was just four colors. In both experiments, however, the maximum color difference between a distractor color and the more similar target was six steps. The analyses below will only include the color-steps that are common across both Target Sets. In other
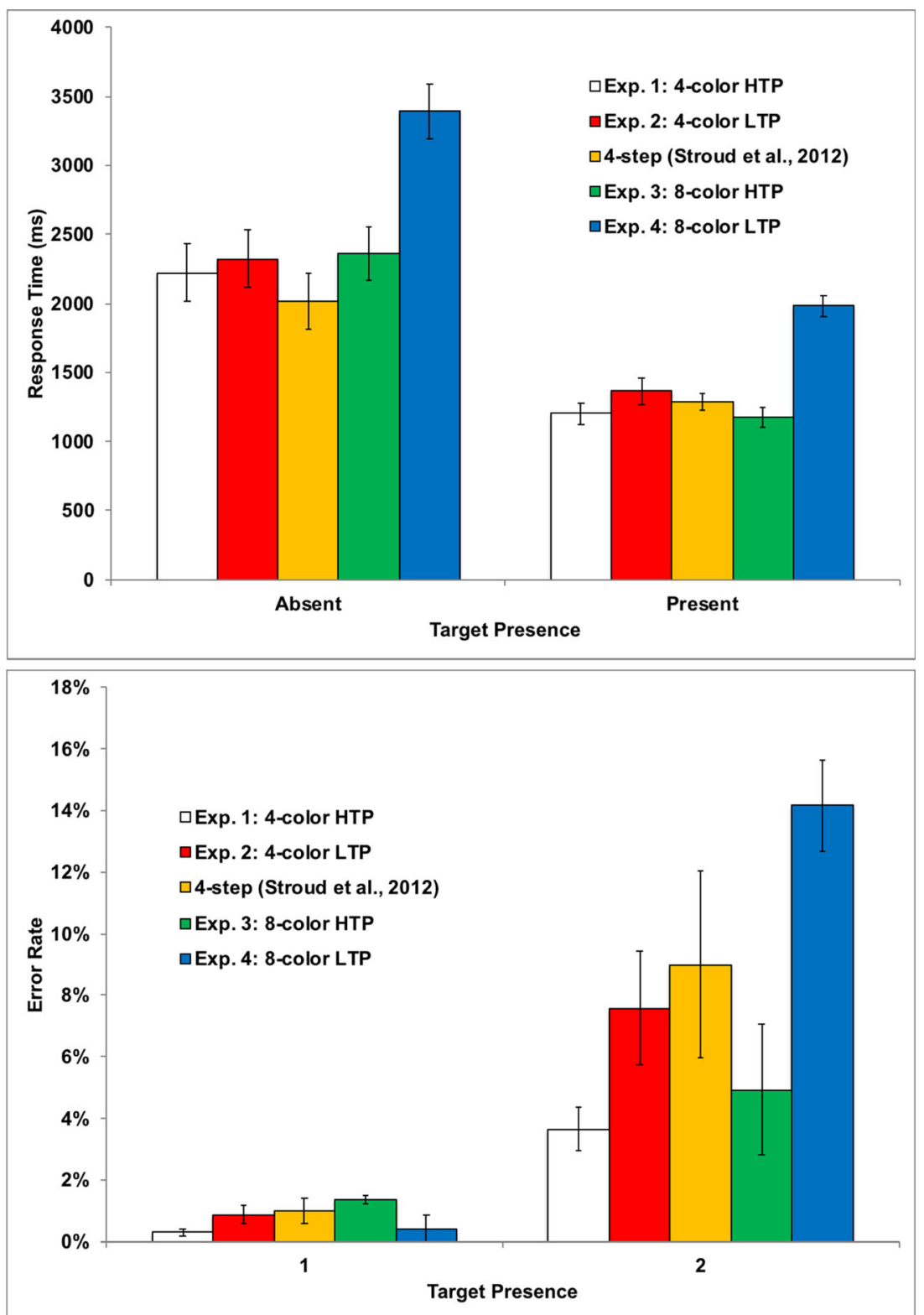

Fig. 4 The response times (top panel) and error rates (bottom panel) for Experiments 1-4. The data from the four-step condition from Stroud et al. (2012) are included as well 
words, only the two target colors at the ends of the target range, and the distractors up to and including six steps from these targets will be included. The resulting probabilities were submitted to a 2 (Target Set) $\times 2$ (Target Presence) $\times 7$ (Color Step) mixed ANOVA with Target Set as the only between-subjects factor. The data are presented in Fig. 5. The effect of Color Step in each experiment is summarized in Table 1.

More fixations were made when the target was absent, $F(1,30)=266, p<.001$, and more fixations were directed towards colors similar to the targets compared to dissimilar, $F(6$,
$180)=120, p<.001$. However, the patterns of fixations were not significantly different between target sets, $F(1,30)=1.410, p=$ .246. The effects of color, the presence of the target, and the search targets were further examined through the interactions.

There was a higher fixation rate for distractors more dissimilar to the target colors when the target was absent compared to when it was present, as reflected through a significant Target Presence $\times$ Color Step interaction, $F(6,180)=15.5, p<$ .001 . This pattern is consistent with a broadening of search, after the target was not initially found, in order to include more
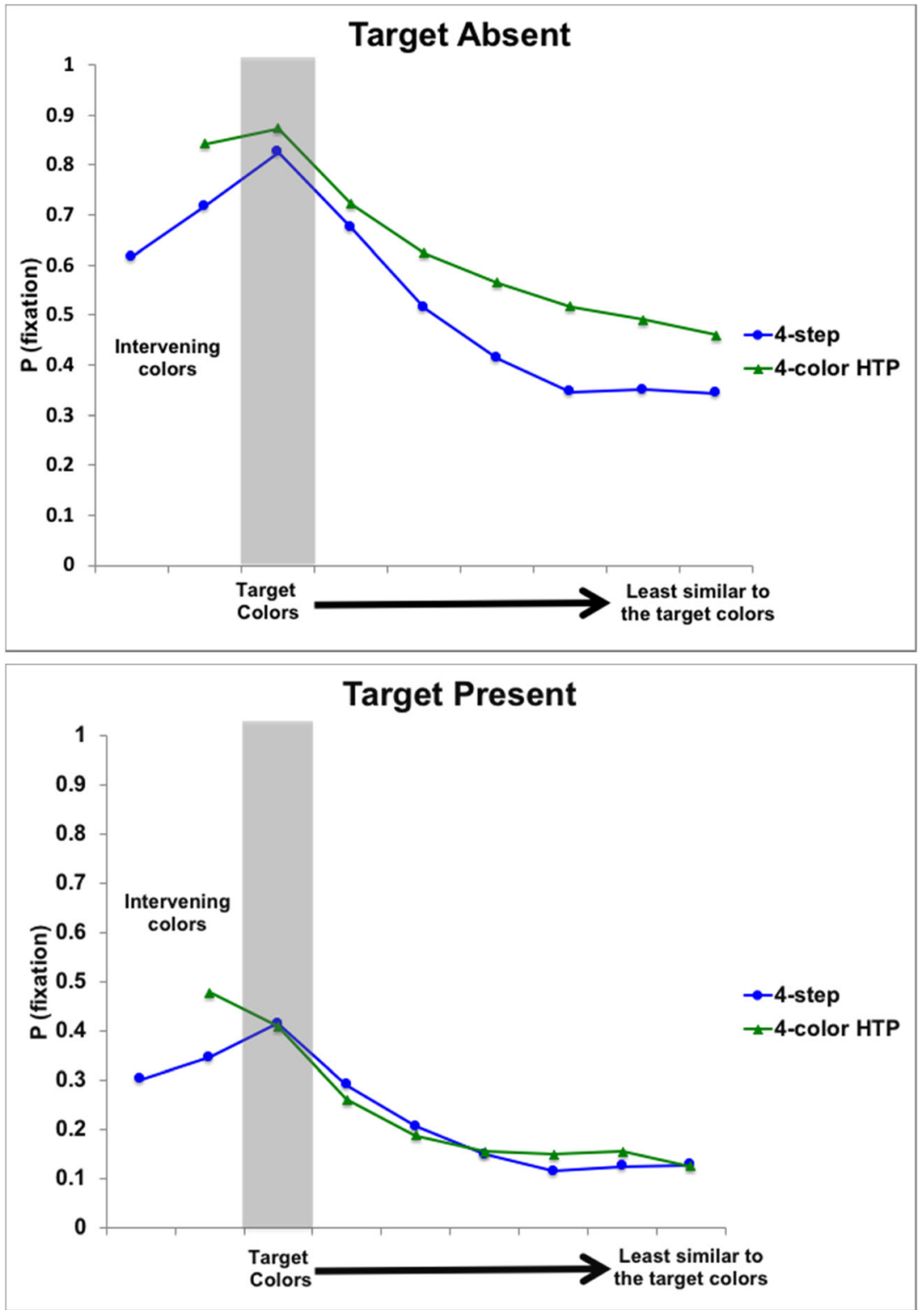

Fig. 5 The fixation probabilities comparing the four-step Target Set, and the four-color High Target Probability conditions from Experiment 1. The top-panel shows results from the target-absent trials, and the bottom panel shows data from target-present. The gray bars indicate the target colors for the four-step condition. In the four-color HTP condition, the colors indicated by the gray bars are the two outermost target colors, and the point to the left of the gray bar represents the two inner target colors. Note that the fixation rates for intervening colors are presented in the graphs, but were not included in the analysis. In the four-step condition, there were three intervening colors represented by the two points to the left of the gray bar, and in the four-color HTP condition, there were only two intervening colors signified by the single point 
colors. There was also a significant interaction between Target Set and Target Presence, $F(1,30)=13.39, p=.014$, but the effect of Target Set was still not significant for either targetpresent or target-absent trials (both $F<2.86, p>.101$ ). .

When considering guidance (i.e., effect of Color Step) across the two Target Sets, the interaction was not significant $(p=.080)$, nor was the three-way interaction $(p=.435)$, providing no evidence for differences in color selectivity between the two Target Sets (see Fig. 5 and Table 1).

\section{Discussion}

There are two striking results from this experiment. First, when target-color items are more likely to be targets than distractors, there is fairly effective guidance by a range of colors that cover $25 \%$ of the hues available. Thus, attentional guidance by multiple colors can be reasonably good when they all come from a single region in color space that includes no non-target colors. The guidance from this fourcolor set does not differ significantly from the guidance shown for the two-target case from Stroud et al. (2012). This pattern suggests that when the representation of four target colors is constructed in the four-color HTP condition, it is just as effective at selecting a target as the two-color representation in the four-step condition. Search for this range of four colors does not appear to require any more resources than the earlier two-target search.

The second finding is that this guidance differs between target-present and target-absent conditions, which may reveal interesting aspects of the time-course of search. The fixation rates for colors very different from the target were much higher when the target was absent than when it was present, replicating the findings of Stroud et al. (2012). This pattern suggests that when a target is not found early in the search, subjects are less certain about the effectiveness of their guidance, and broaden their search to fixate more distractors before terminating.

A factor that may be strengthening the target representation in the four-color HTP condition, despite the need to represent four targets, is the information accumulated over time regarding the probability that a target color is actually a target. Recall that in this experiment, when an object is present in the color of the target, it is more likely to be a target than a distractor. Thus, when the target color appears, there is more motivation to attend to it than in the four-step condition (Stroud et al., 2012). The need to separate target probability from other factors motivated Experiment 2, which was identical to Experiment 1, except that the distractor colors were selected such that the target-colored objects were more likely to be distractors than targets, as in the four-step condition (Stroud et al., 2012). If this bottom-up information is influencing search guidance, then we should see a marked difference in the fixation patterns between the Experiments 1 and 2.

\section{Experiment 2}

The results of Experiment 1 demonstrated that it is possible to direct attention to any of four colors within a range that covers $25 \%$ of the hue dimension. How critical is it, though, that the target colors within the displays be more indicative of a target than a distractor? The purpose of the current experiment is to replicate Experiment 1, but with the probability that a targetcolor item is a target set to be the same as in Stroud et al. (2012). That is, when a target color appears, it is more likely to be a distractor than a target.

\section{Method}

\section{Subjects}

Sixteen additional subjects recruited from the University of Massachusetts community took part in Experiment 2. The subjects had a mean age of 19.9 years $(S D=1.2)$.

\section{Design}

The new subjects were assigned to the four-color low target probability (LTP) condition, and their results were compared against the four-color HTP condition of Experiment 1. Thus, three factors were manipulated in the current experiment resulting in a 2 (Target Color Probability ${ }^{1}$ : four-color HTP vs. four-color LTP) $\times 2$ (Target Presence: Absent vs. Present $) \times 8$ (Color Step) mixed factorial design with Target Set as the only between-subjects factor. Color step in this case included two levels for the target colors (inner and outer), and six levels for non-target colors.

\section{Apparatus and stimuli}

The same materials and equipment were used as in the previous experiment. A new set of stimuli was generated to match the specific target/distractor probabilities. As in Experiment 1, there were four target colors, giving eight targets of each color at each orientation across the 128 target-present trials. In the current experiment, we matched the probability that a targetcolored item is actually a target with that in the four-step condition. In order to achieve that target probability, we used 19 of each target-color distractor (i.e., each unique color/ orientation combination) and 44 or 45 of each non-targetcolor distractor. Target-colors items were therefore targets $30 \%$ of the time, as in Stroud et al. (2012).

\footnotetext{
${ }^{1}$ In this experiment, Target Color Probability represents the target/distractor probabilities rather than a difference in search targets.
} 


\section{Procedure}

The procedure was identical to Experiment 1.

\section{Results and discussion}

Error rates and RTs were submitted to a 2 (Target Color Probability) $\times 2$ (Target Presence) ANOVA. When the target was present, subjects committed more errors, $F(1,30)=31.32$, $p<.001$ and responded faster, $F(1,30)=99.97, p<.001$, compared with target-absent trials. When comparing the two Target Color Probability conditions, subjects were more accurate in the four-color HTP than the four-color LTP condition, $F(1,30)=4.18, p=.05$ (see Fig. 4).

The fixation probabilities were submitted to a 2 (Target Color Probability) $\times 2$ (Target Presence) $\times 8$ (Color Step) repeated measures ANOVA. There were main effects of Target Presence, $F(1,30)=564, p<.001$ and Color Step, $F(7,210)=$ $103, p<.001$, revealing fewer fixations when the target was present and to distractor colors relative to the target colors, respectively. There were no significant main effects or interactions involving Target Color Probability, reflecting no influence of target probability on search (see Fig. 6 and Table 1).

Figures 5 and 6 show a general pattern in the fixation rates that suggests strong guidance towards the colors in the middle of the target range, and a gradual decline for colors less and less similar to these central colors. One possible explanation is that in Experiments 1 and 2, the target representation includes just a single color at the center of the target range, and that it generates a gradient of attentional activation across a broad region of color space. Such a strategy would be consistent with Navalpakkam and Itti's (2007) claim that the most effective target representation for guiding search does not necessarily precisely reflect the target colors.

The gradual decrease in fixation with color similarity is also consistent with Schönhammer, Becker, and Kerzel's (2017a) broad attentional tuning account that explains the results of their Experiment 2. That pattern contrasts with the data from their Experiment 1, and with their other experiments (Becker, 2010; Becker, Folk, \& Remington, 2013; Schönhammer, Grubert, Kerzel, \& Becker, 2017b) indicating that attention is often directed not to a specific color, but to the color that has a specific relation (i.e., redder) to the other colors in the display. The stimulus colors in those experiments were usually taken from a fairly narrow range that forms a line in color space. In the new experiments presented here, the stimulus colors include many different hues, and the pattern of similarities across these different hues forms a circle. This arrangement makes it difficult to specify the target colors in relational terms. Thus, if there is a possibility of specifying target information in either relational or absolute terms, as suggested by the differing results between Schönhammer, Becker, and Kerzel's two experiments, then the color ring used in these experiments might be expected to promote a representation of absolute target colors. Schönhammer, Becker, and Kerzel (2017a) note that the results from their relational experiments might also be explained by a broadly tuned attentional setting centered on a color at the extreme end of their stimulus range. Thus, it is possible that the same broad target representation might be used in Becker's experiments and in our Experiments 1 and 2.

The frequency with which a color appears as a target does not affect how that color is used to guide eye movements in this experiment, although the accuracy data suggest that the frequency can affect the likelihood that an item is labeled as a target after it is attended. It would be an over-generalization, however, to conclude that a color's target frequency never affects search guidance, as is shown in the following two experiments. The next experiment will test whether the set of search targets can be extended further; in Experiment 3 it will include half of the available hues.

\section{Experiment 3}

The results of Experiments 1 and 2 revealed that it is possible to guide search for four target colors that are taken from adjacent positions on the stimulus color ring when targets with all four of those colors appear in the search task. It is not necessary that the target colors themselves be a very reliable cue to the target presence, as there was no difference in the fixation patterns between the two experiments. The purpose of Experiment 3 is to examine if an expanded set of eight target colors (half of the hues in the stimulus color space) can be represented in order to effectively guide search. In this first experiment with eight target colors, the probability that a target color will actually appear as a target was set to be the same as in Experiment 1 (the high target probability, four-color HTP, condition).

\section{Method}

\section{Subjects}

Sixteen additional subjects recruited from the University of Massachusetts community took part in Experiment 3. The subjects had a mean age of 20.0 years $(S D=1.2)$.

\section{Design}

All subjects in this experiment were assigned to the eight-HTP condition, producing a 2 (Target Presence: Absent vs. Present) $\times 8$ (Color Step) mixed factorial design. Color step included four levels for the target colors (one to four steps from the most similar non-target color), and four levels for the non-target colors (one to four steps from the most similar target color). 


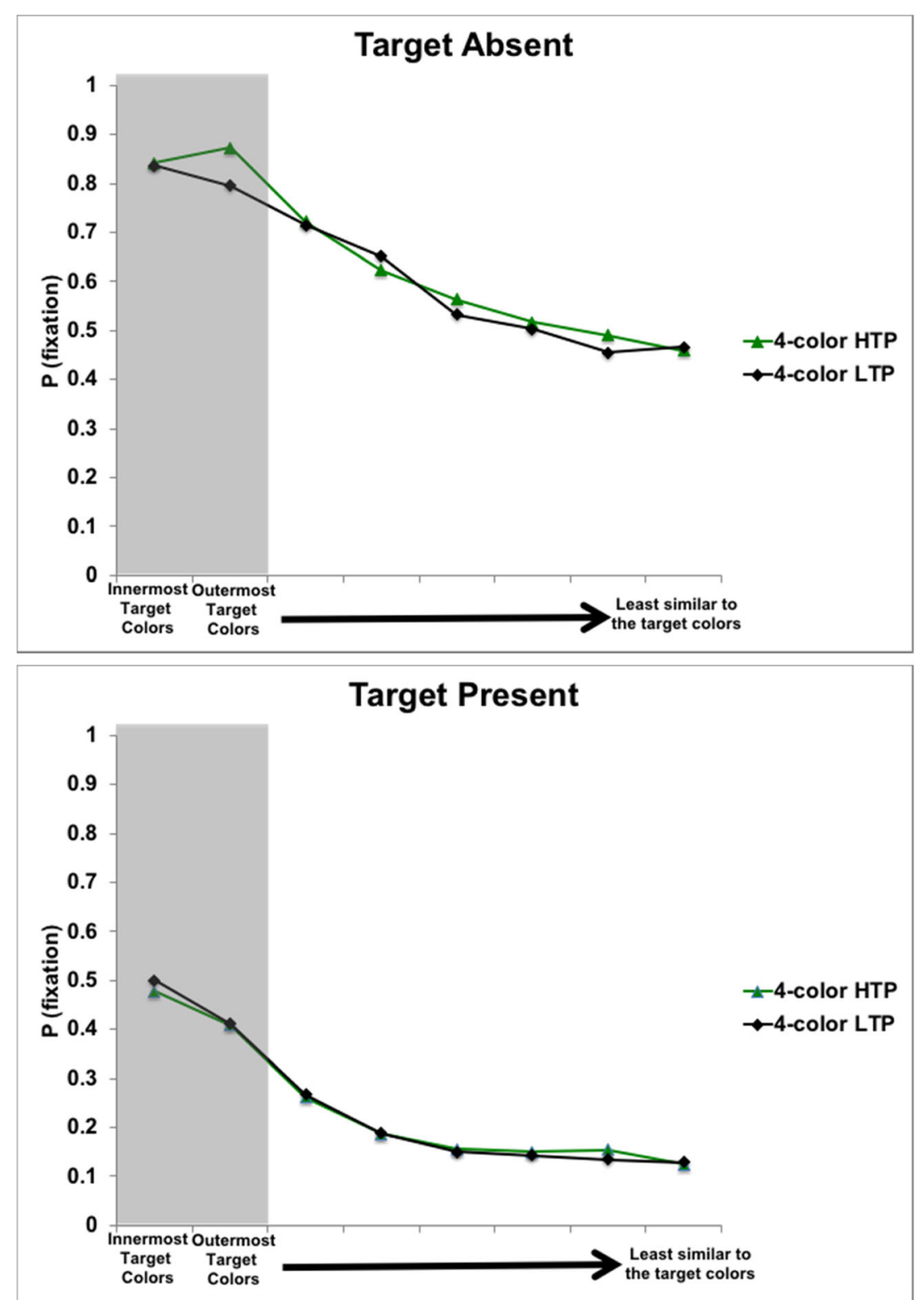

Fig. 6 The fixation probabilities for the four-color HTP (Experiment 1) and the four-color LTP (Experiment 2) Target Color Probabilities. The top panel is for Target Absent and the bottom panel is for Target Present

\section{Apparatus and stimuli}

The equipment and settings from the previous experiments were used for the current experiment. The stimuli were designed similarly to previous experiments, except that the targets could appear in eight possible colors that occupied adjacent positions on the 16-color ring of possible colors. The same number of trials was included as in the previous experiments, with each target color appearing an equal number of times (four times each at each orientation). Instead of a four-color patch preview, subjects were provided with patches of eight colors before each trial (see Fig. 7). The stimuli were created such that the probability that a target-color item was a target was the same as that used in the four-color HTP condition (Experiment 1). There were 73 or 74 of each non-target-color distractor (at each orientation) and two or three of each target-color distractor. Once again, this resulted in the target colors appearing more frequently as targets than distractors, with a probability of .62. As before, target colors were balanced across subjects.

\section{Procedure}

The same exact procedure was used as Experiments 1 and 2. Again, each subject viewed a different set of eight targets so

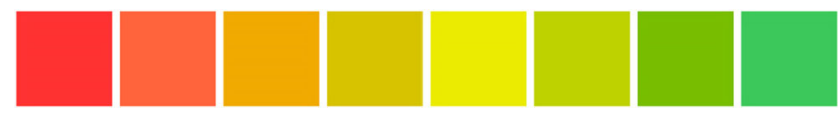

Fig. 7 An example of the eight-color patch preview 


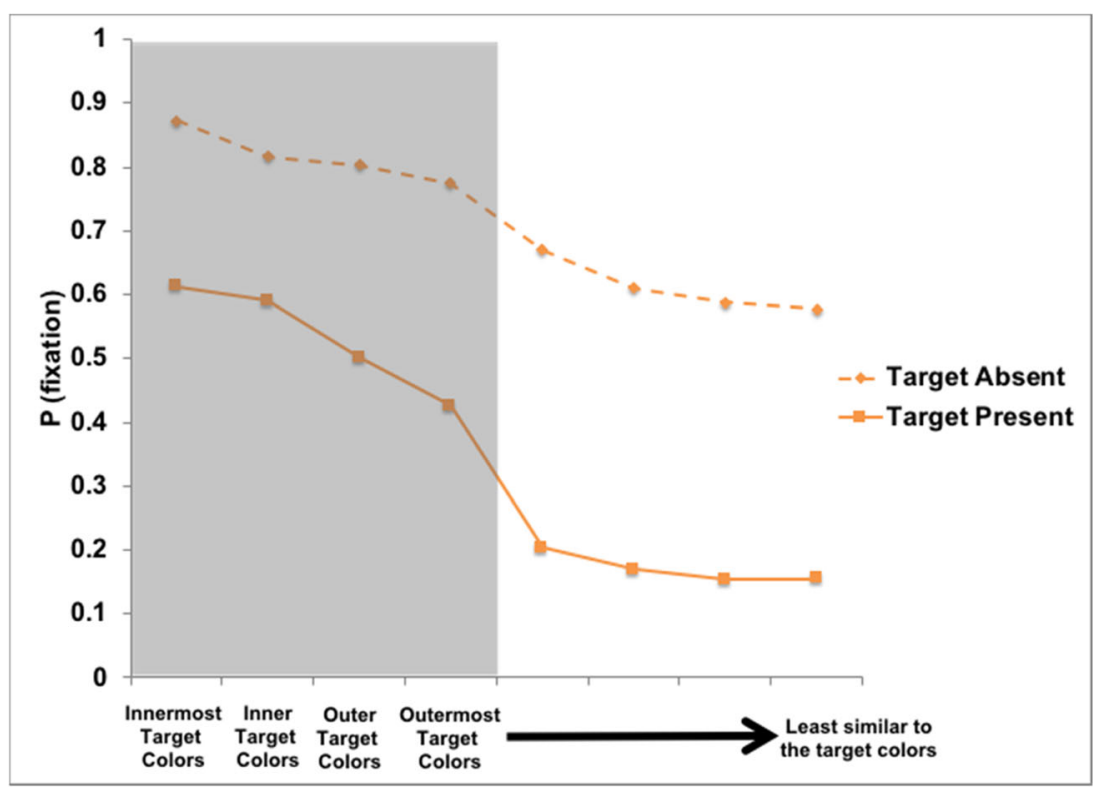

Fig. 8 The fixation probalities for the eight-color HTP (Experiment 3) Target Set

that all combinations of colors were equally represented across the sample.

\section{Results and discussion}

The main purpose for the current experiment was to test for evidence of color selectivity with eight target colors. We encouraged subjects to use color to guide search by creating a high probability that a target-color item would be a target. Subjects responded faster, $t(15)=8.23, p<.01$, and committed more errors, $t(15)=2.26, p=.039$, when the target was present compared to when it was absent. The fixation probabilities were submitted to a 2 (Target Presence) $\times 5$ (Color Step) repeated measures ANOVA. Only the outermost targets were included with the non-target colors in the analysis. See Fig. 8 for fixation rates for both target-absent and targetpresent trials.

The results produced main effects for both Target Presence, $\mathrm{F}(1,13)=98.64, \mathrm{p}<.001$, and Color Step, $F(7,91)=29.32, p$ $<.001$. A significant interaction between the two factors, $F(7,91)=3.044, p=.006$, reflected a stronger color guidance when the target was present. The main finding is that a reasonable level of guidance can be achieved spanning eight colors (i.e., half of the colors available), with the caveat that the target colors are reliable cues to target identity.

In this experiment, as in the earlier experiments, each subject searched for a different set of target colors, with each color serving as a target equally often. Some target sets may have allowed for more effective guidance than others, and some sets probably contained more color category boundaries than others, which might have determined the difficulty of representing target colors and guiding search. Thus, some target color sets may produce better guidance than others, but the results averaged across these different subjects nonetheless demonstrate that a reasonable level of search guidance is possible with a very broad range of different colors. This issue will be explored further in Experiment 4. ${ }^{2}$

If the target template for this task is stored in working memory, which has a very limited capacity, then the eight targets are probably not represented in the search template as eight separate objects, but as a unified template representing the range of target colors. If such a range representation is possible, then why was it not used in Stroud et al.'s (2012) four-step color search? As discussed in the Introduction, some of Stroud et al.'s subjects did show evidence of using a range representation, as indicated by a high fixation rate for colors that were in between the two specified target colors in color space. However, the overall pattern of fixations, across all subjects, recorded during those searches indicates that generally the two targets were represented separately.

The graph in Fig. 8 suggests that guidance might be somewhat better for colors near the middle of the target range than for colors at the edge. However, it also seems to show a strong drop-off between the target-color and non-target-color regions, at least in the target-present trials, suggesting that the target representation for this broad range does not consist of just a single color at the center of the range.

Thus far, the results of Experiments 1-3 demonstrate that four colors ( $25 \%$ of the available hues) and eight colors (50\% of the hues) can be represented to guide search. However, it is possible that guidance by this large set of eight colors is sensitive to the increased target probabilities, even though guidance by four colors was not. Experiment 4 was designed to test for an effect of target probability under these more extreme conditions.

\footnotetext{
${ }^{2}$ The authors would like to thank Jeremy Wolfe for this observation.
} 


\section{Experiment 4}

The results of Experiment 3 showed that color selectivity and search guidance are possible with eight target colors. Previously, in comparing Experiments 1 and 2 (four-color HTP and four-color LTP respectively), we found that guidance did not appear to be sensitive to the probability that a target-colored item would be a target. If search guidance is going to be adjusted according to target probability, then it would seem more likely that it would happen when there is a large number of target colors. Therefore, in order to test again whether this target probability can have an effect, we repeated Experiment 3 (eight-color HTP), but used the same frequencies of distractors as in Stroud et al.'s (2012) four-step condition, which resulted in an extremely low target probability, and thereby provided a large difference from the high target probability used in Experiment 3 (eight-color HTP).

\section{Method}

\section{Subjects}

Sixteen additional subjects recruited from the University of Massachusetts community took part in Experiment 4. The subjects had a mean age of 19.9 years $(S D=1.5)$.

\section{Design}

The new subjects were assigned to the eight-LTP condition, and their results were compared against those of the eightcolor HTP condition in Experiment 3. Thus, the current experiment employed a 2 (Target Color Probability: eight-color LTP vs. eight-color HTP) $\times 2$ (Target Presence: Absent vs. Present $) \times 8$ (Color step) mixed factorial design with Target Color Probability as the only between-subjects factor. Color step in the current experiment included four levels representing the eight target colors and four levels representing the eight non-target colors, as it did in the previous experiment.

\section{Apparatus and stimuli}

The current experiment was identical to Experiment 3 except that the frequencies of the different distractor colors were changed such that the probability that an item with a target color was actually a target was decreased. Similar to the fourstep condition of Stroud et al. (2012), each color appeared as distractors with equal frequency ( 38 of each at each orientation). However, with eight target colors, each target is presented four times, giving a target probability of just $9.5 \%$.

\section{Procedure}

The procedure was the same as the previous experiments.

\section{Results and discussion}

Accuracy and response times were submitted to a 2 (Target Color Probability) $\times 2$ (Target Presence) repeated measures ANOVA. Responses were slower and more accurate when the target was absent versus present $(F(1,30)=161, p<.001$ and $F(1,30)=43, p<.001$, respectively), as expected. There was no difference in accuracy between the Target Color Probability conditions when the target was absent, but subjects committed more errors when the target was present in the eight-color LTP (mean difference 10\%) resulting in a significant Target Color Probability $\times$ Target Presence interaction, $F(1,30)=14.8, p=.001$. Responses in the eight-color HTP were significantly faster than the eight-color LTP, $F(1,30)=$ $595, p<.001$, without an interaction with Target Presence. Taken together, the accuracy and response time data appear to reflect a slightly easier task when the target colors were informative of the targets (see Fig. 4).

The fixation probabilities were submitted to a 2 (Target Color Probability) $\times 2$ (Target Presence) $\times 8$ (Color Step) repeated measures ANOVA. Figure 9 presents the data. There were three main effects, which showed that more objects were fixated when the target was absent versus present, $F(1,30)=469, p<.001$, when the color was similar to the target compared with dissimilar, $F(7,210)=32, p<.001$, and when the target colors were more likely to appear as distractors (eight-color LTP vs. eight-color HTP), $F(1,30)=$ $12.2, p=.001$. This last effect of Target Color Probability reflects the lack of guidance for eight target colors when color is not very informative about the target (see Table 1).

Target Presence and Color Step significantly interacted, because color selectivity was stronger (i.e., the difference in fixation rates between target and non-target colors was greater) when the target was present compared to when it was absent, $F(7,210)=3.81, p=.001$. What is perhaps most revealing are the various significant interactions that clearly demonstrate that use of color was directly affected by statistical probabilities of the target colors (see Fig. 9). Color selectivity was present in the eight-color HTP condition (Experiment 3) but it was completely absent in eight-color LTP, resulting in a significant Target Color Probability $\times$ Color Step interaction, $F(7,210)=26, p<$ .001 . There was also a significant interaction between Target Color Probability, Target Presence and Color Step, $F(7,210)=$ $4.02, p<.001$, because selectivity was higher for target-present in eight-color HTP while selectivity was nonexistent regardless of target presence in eight-color LTP.

Figures 8 and 9 show that fixation rates are quite constant across target and non-target colors in the low target probability (LTP) condition. This pattern indicates that effective guidance 

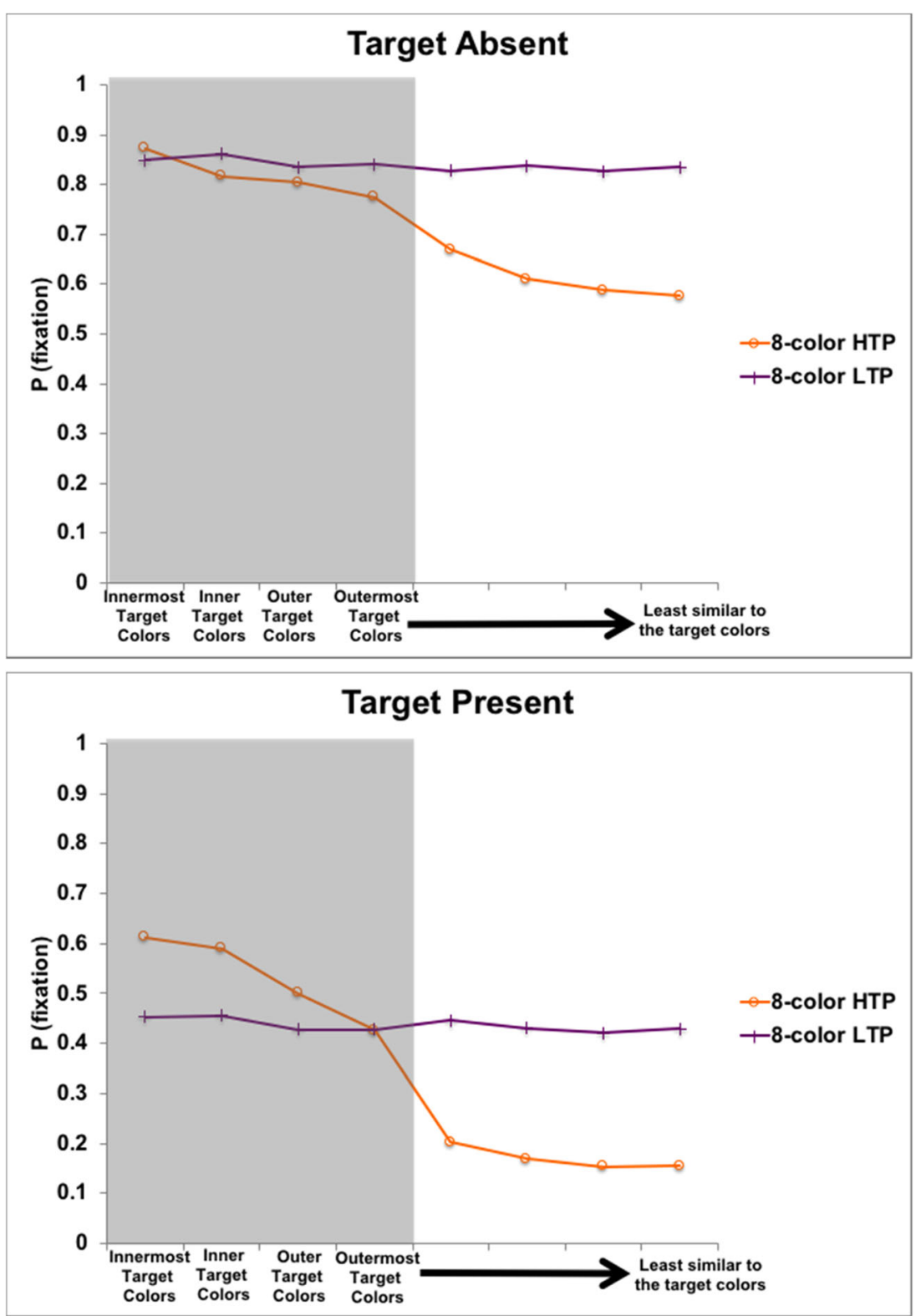

Fig. 9 The fixation probabilities for the eight-color HTP (Experiment 3) and eight-color LTP (Experiment 4) Target Color Probabilities with the target absent in the top panel and the target present in the bottom panel

does not occur when the target set includes half of the color hues and a target-colored item has a very low probability of being a target. Such a low target probability, combined with the large number of objects held in WM, may have been too much for the system to handle. This strong effect of target probability contrasts with the comparison of Experiments 1 and 2 (four-color HTP and four-color LTP), in which target probability had little effect. Experiment 4 was designed to push the system to the limits with a large set of target colors to test whether target probability can ever affect guidance, and in this case guidance was eliminated when target-colored items were unlikely to be targets. Given that subjects have the ability to guide search by the eight-color target set, as demonstrated by the decrease in fixation rates between target and non-target colors in Experiment 3, it is surprising that subjects do not make effective use of this guidance in Experiment 4. Even though this guidance would have selected a large number of target-color distractors, it would have prevented fixations to a large number of non-target-color distractors, and thus made search more efficient. For some reason, the large set of target values combined with the low target probability made it less likely that search was guided by color. Either there is a significant cost in maintaining a large target set representation that offsets the advantage of avoiding numerous fixations to distractors, or subjects are failing to deploy search guidance as effectively as they could.

Despite the lack of guidance under the circumstances in the current experiment, Experiments 1-3 showed that participants 
are able to search for multiple target colors that all come from one specific region of color space that includes no non-target colors. In addition, it seems plausible that guidance for eight target colors (Experiment 3, eight-color HTP) was facilitated by combining the target colors into a unified representation, rather than maintaining eight discrete representations. The differences in the results across these experiments demonstrates that subjects have flexibility in how they guide search. The remaining experiments will test whether subjects can be encouraged to use a single unified target template under the dualtarget search conditions used in earlier experiments.

As mentioned above, the eight-color target sets used in Experiments 3 and 4 span across more color category boundaries than the four-color target sets in Experiments 1 and 2, and the larger target sets may result in more variation in color category boundaries across participants. The target color sets are assigned to participants in order to balance these differences: each set of eight target colors is used once across the 16 participants. Averaging over all participants minimizes the impact of qualitative (e.g., color categories) and quantitative (e.g., color distance) differences across target sets on the findings. There are also individual differences in color perception (Neitz \& Jacobs, 1986) and there has been much debate over the effect of color categories on speed of distinguishing between colors (e.g., see Forder, He, \& Franklin, 2017; Webster \& Kay, 2012). It is would therefore be difficult to establish control over color category boundaries when using the desired number of colors employed in the current experiments. However, it is not clear how such differences would account for the current findings. Experiments 1-4 revealed that target set size interacts with the informativeness of the target color to determine whether search is guided by color. Given that stimulus color variation exists in all conditions, it seems unlikely that it would produce such an interaction.

\section{Experiment 5}

The effective guidance in Experiments 1-3 suggests that Stroud et al.'s subjects ought to have been able to guide their searches with a set of colors that included both target colors. Perhaps subjects will adopt such a search strategy if they are encouraged to think of the target set as a range of colors. Experiment 5 tested whether a change in the instructions is enough by itself to prompt subjects to change the way they represent the target colors. It utilized the exact same stimuli from the four-step Target Set in Stroud et al., (2012), but Experiment 5's subjects were shown a single stimulus depicting a range of possible target colors, and asked to search across the entire range instead of searching for just the two separate discrete target colors. If subjects did search for the entire range of colors, fixation rates should be high to the target colors and the three intervening colors. Because the

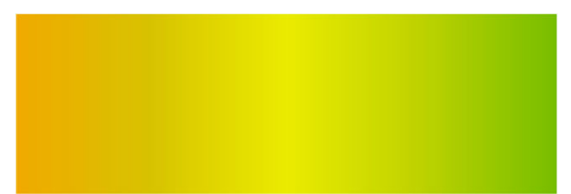

Fig. 10 An example of the target range stimulus specifying the possible target colors for Experiment 5. This range corresponds to the targets from Stroud et al. (2012) for the four-step Target Set. Even though subjects were presented with the entire range, the targets only appeared in the two outermost colors

same search arrays and targets were used as in Stroud et al., the targets that appeared in the search arrays had one of the two colors on either end of the range stimulus (see Fig. 10). Subjects were not informed that only the colors at the ends of the range appeared as targets, but were asked a number of questions regarding their interpretation of the target range after completing the experiment.

There are a number of possibilities for how subjects will perform in the current experiment. If subjects can actually represent a range of colors including the two targets, then fixation rates to the three intervening distractor colors should be high and comparable to rates for the targets, while fixation rates to colors outside this range should remain as low as those observed in four-step dual-target search. On the other hand, search may be guided by the two outside colors of the range (which are the only colors that ever appear as targets) and the three intervening distractor colors might be ignored. A third option is that encouraging a range representation interferes with color selectivity altogether, which would reveal a lack of guidance towards target colors and potential target colors.

\section{Method}

\section{Subjects}

Sixteen University of Massachusetts students, with ages ranging from $18-24$ years $(M=20.9, S D=1.8)$ took part in Experiment 5.

\section{Design}

The design was similar to the previous experiments. The new subjects each received a target preview comprising a range of colors for comparison with the four-step condition from Stroud et al. (2012). The resulting design was a 2 (Target Set: four-step ${ }^{3}$ vs. four-step range) $\times 2$ (Target Presence: absent vs. present) $\times 9$ (Color Step) mixed factorial design with Target Set as the only between-subjects factor. Color step included one level for the two target colors, two levels for the three colors in between the target colors, which were within

\footnotetext{
3 The data from the four-step Target Set are those from the four-step condition in Stroud et al. (2012). It is important to note that the two targets in this condition were discrete search targets.
} 
the color range depicted by the rainbow stimulus, and six levels for the non-target colors.

\section{Apparatus and stimuli}

The current experiment utilized the same equipment and search objects as the previous experiments. Each unique color/orientation of distractor was presented 38 times, resulting in the same target-color relationships as Stroud et al. (2012). Even though participants were instructed to search for an entire range of colors, half the targets had the color at one end of the range, and half had the color at the other end, with no targets having colors from the middle of the range. The targets were therefore the same two colors as used in Stroud et al. (2012).

The search targets for a given subject consisted of the same two colors throughout the entire experiment, with the two colors being four steps apart in stimulus color space. As in the previous experiments, a target was present on $50 \%$ of the trials, with targets in each of the colors appearing in the same number of trials, and the target colors balanced across subjects.

In the experiments reported by Stroud et al. (2012), the two possible target colors were presented to the subject as two separate discrete objects, each with a uniform color. In the current experiment, subjects learned of the target colors by viewing a single long bar within which the hue smoothly changed from one end to the other to specify a range of colors from a contiguous region of color space. Because of the gradual color change this stimulus resembled a portion of a rainbow or color spectrum, and thus we will refer to it as a rainbow stimulus. The two target colors that would actually appear were represented at the left and right ends of the stimulus. The target range stimulus subtended roughly the same visual angle as the two-object color preview from Stroud et al. An example target cue, for one target-color pair is shown in Fig. 10.

\section{Procedure}

The experiment followed the same procedure as in Stroud et al. (2012), except for what subjects were told about which target colors to expect, and how those target colors were depicted in the instructions. In the new experiment, they were told to expect that the $\mathrm{T}$ could be any of the target colors within the range stimulus presented. Also, after the experiment was completed, subjects were asked a number of questions to see if they were able to determine that the targets only appeared as the outside colors of the range stimulus. Subjects were shown the target range and asked the following questions: (1) Which colors did the T appear as? (2) Did you devise a strategy for searching? (3) Did you ignore any part of the range and search for specific colors? If subjects had trouble divulging a search strategy, then further questions were directed towards asking about specific uses of color, areas of the range and so on.

\section{Results}

Most subjects reported that the targets mostly appeared towards the end of the range, but not specifically the outer edge. Despite the fact that the majority of subjects made this observation, no subject reported altering his or her strategy for searching throughout the entire experiment. Some went as far to express that they "felt as though the target would eventually appear in the other colors."

\section{Accuracy and response times}

Average response times and error rates (see Fig. 11) were submitted to a 2 (Target Set: four-step vs. four-step range) $\times 2$ (Target Presence: absent vs. present) ANOVA with Target Set as a between-subjects factor. In Experiment 5, subjects were less accurate and responded faster when the target was present rather than absent. There was no significant difference between four-step and four-step range (mean difference $=.0008$ ) for error rates. Subjects responded faster with the four-step Target Set $(1,747 \mathrm{~ms})$ compared with the four-step range Target Set $(2,219 \mathrm{~ms}), F(1,30)=9.00, p=.005$. However, the difference in RT between Target Sets was greater when the target was absent, as revealed through a significant Target Set $\times$ Target Presence interaction, $F(1,30)=6.73, p=.015$.

The fixation rates are presented in Fig. 12. The fixation rates were submitted to a 2 (Target Set) $\times 2$ (Target Presence) $\times 7$ (Color Step) repeated measures ANOVA. Consistent with Stroud et al. (2012), subjects were color selective and fixated more objects when the target was absent, as revealed through main effects of Color Step, $F(1,30)=64, p<.001$ and Target Presence, $F(1,30)=376.28, p<.001$, respectively. Subjects presented with the range target specification fixated significantly more objects than those presented with two separate target objects, $F(1,30)=12.10, p=.002$. The range depiction of the target colors raised the fixation rate more for the target-absent condition than for the target-present, resulting in a significant Target Set $\times$ Target Presence interaction, $F(1,30)=7.90, p<$ .001 . These patterns are consistent with the response time data. Color step and Target Presence significantly interacted, $F(8,240)=7.68, p<.001$, again replicating Stroud et al.'s (2012) findings that subjects fixated fewer objects and were more color selective when the target was present.

Seeing the target colors as part of a range dramatically altered subjects' use of color, evidenced by a significant Color Step $\times$ Target Set, $F(8,240)=13.28, p<.001$ interaction. Overall, subjects were less selective about which colors they fixated when subjects were given the target range; in other words, the increase in fixation rate from seeing the targets as a range was especially strong for colors very different from the targets. This drop in selectivity was stronger when the target was absent, resulting in a significant Target Set $\times$ Target Presence $\times$ Color Step, $F(8,240)$ $=10.85, p<.001$, interaction. 


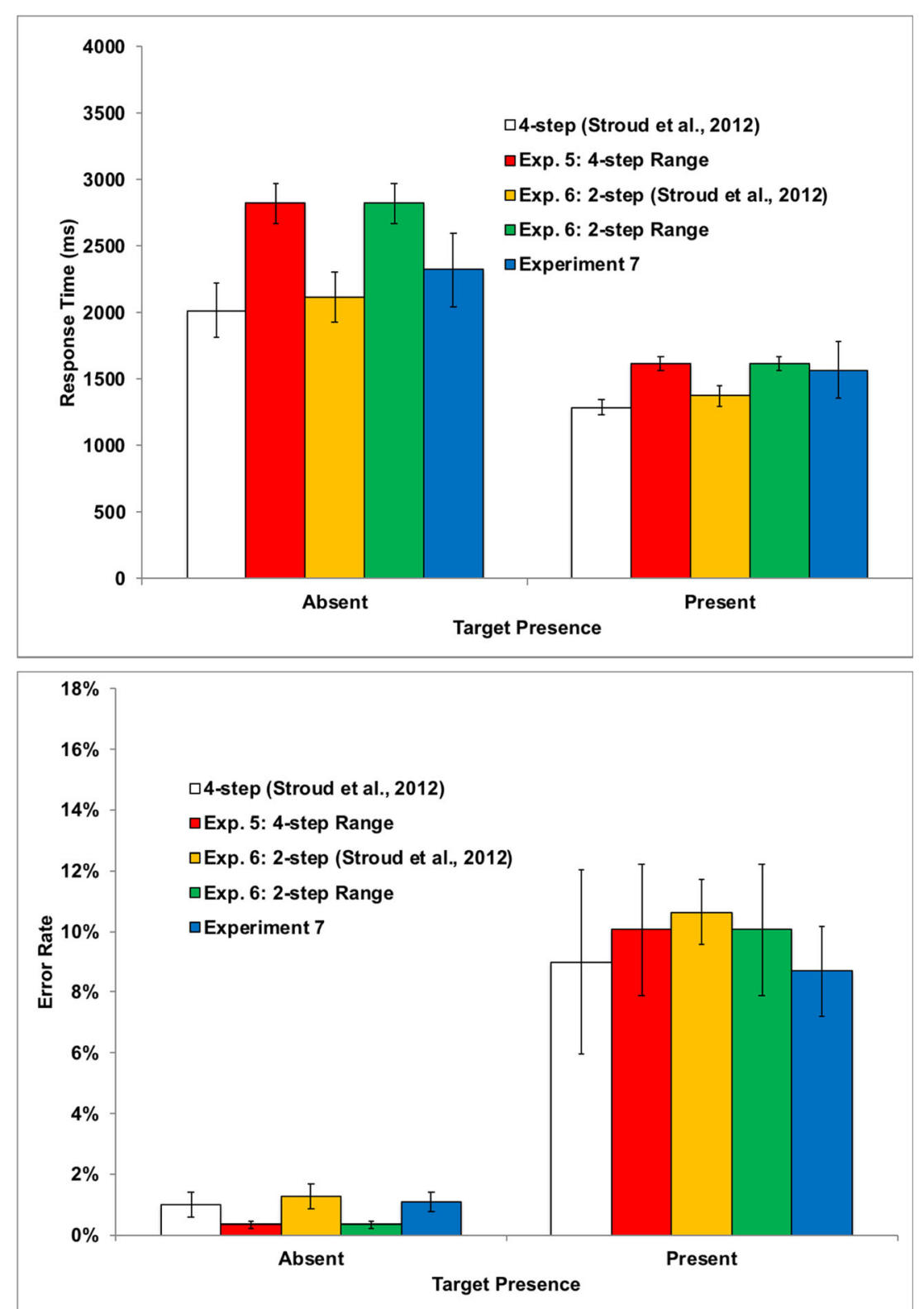

Fig. 11 The response times (top panel) and error rates (bottom panel) for Experiments 5-7. Once again, the data from the four-step condition from Stroud et al., (2012) are included for comparison

The original aim of this experiment was to examine the fixation rates to the colors that fall between the two target colors in stimulus color space; that is, the intervening colors that are presented to the subjects in the four-step range condition. Planned comparisons conducted on these intervening distractor colors revealed that subjects fixated significantly more of them when given the four-step range than the four-step targets, both when the target was present, $t(30)=2.40, p=.023$, and when the target was absent, $t(30)=2.41, p=.022$ (Fig. 12).

\section{Individual differences}

As in Stroud et al.'s analysis, the individual subject data were inspected for the four-step range Target Set to assess the degree to which subjects may have utilized a broad, unitary range versus two discrete target templates. The fixation probability for the middle intervening color was subtracted from the mean of the two target colors to yield a difference score, so that positive values indicate that the target colors receive more fixations than the intervening color. We focused the analysis on the middle color only because it is the distractor color that is most likely to show a difference compared to the targets. These difference scores for the four-step range are plotted along with the scores from the four-step Target Set in Fig. 13 to provide a direct comparison between the two Target Sets.

Given that the pair of target colors that appeared in the displays was different for each set, it is not surprising that fixation patterns varied across subjects within a condition. 

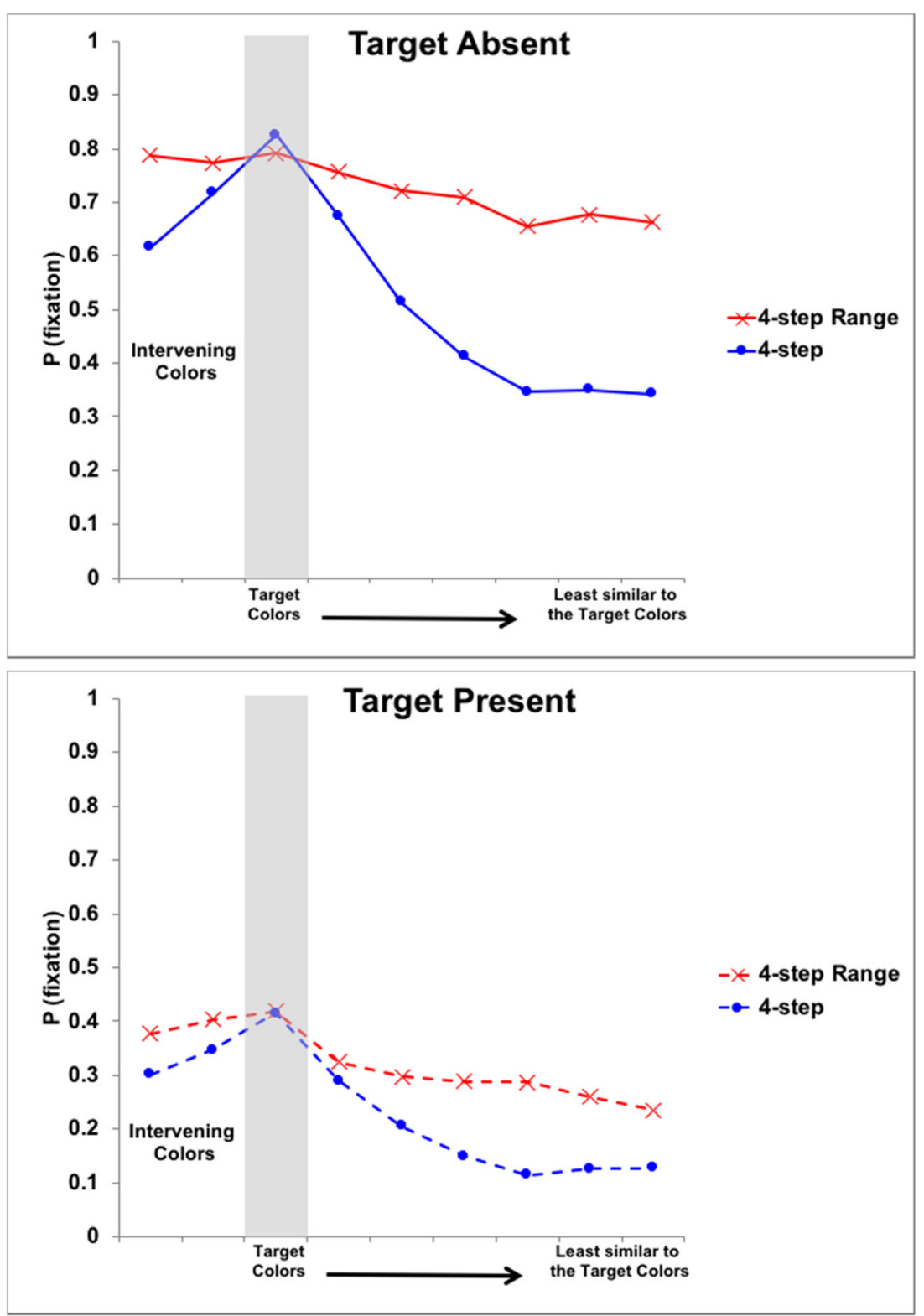

Fig. 12 The probability of fixation for the four-step and four-step range Target Sets in Experiment 5 when the target was absent (top panel) and when the target was present (bottom panel). The gray bar indicates the target colors in the four-step condition, which correspond to the colors at

However, the same sets of target pairs were used in both the discrete and range conditions, and thus the differences across the two conditions are potentially informative. For the discrete Target Set, as reported in Stroud et al. (2012), seven and 12 of the 16 subjects fixated target-color distractors significantly more often than the intervening colors on target-present and target-absent trials respectively (as determined by $95 \%$ confidence intervals for the differences). For the range Target Set, these values were two and one out of 16 subjects, respectively. The comparison shows less difference between target colors and the intervening color when the target was depicted by a rainbow stimulus, but this may be a consequence of the the two ends of the target color range shown to the subjects in the fourstep range condition. Note that as in the analysis, fixations to targetcolored distractors are included in the graphs, but fixations to targets are not

fixation rates for all non-target colors being higher in the range condition.

\section{Discussion}

Experiment 5 investigated whether subjects could utilize a range of target colors as an effective representation for guiding search when the target color was specified by a rainbow stimulus. It was suspected that the rainbow stimulus presented to the subjects to specify the target colors would produce the greatest increase in fixation rates for the three intervening colors that are between the two targets in color space because 


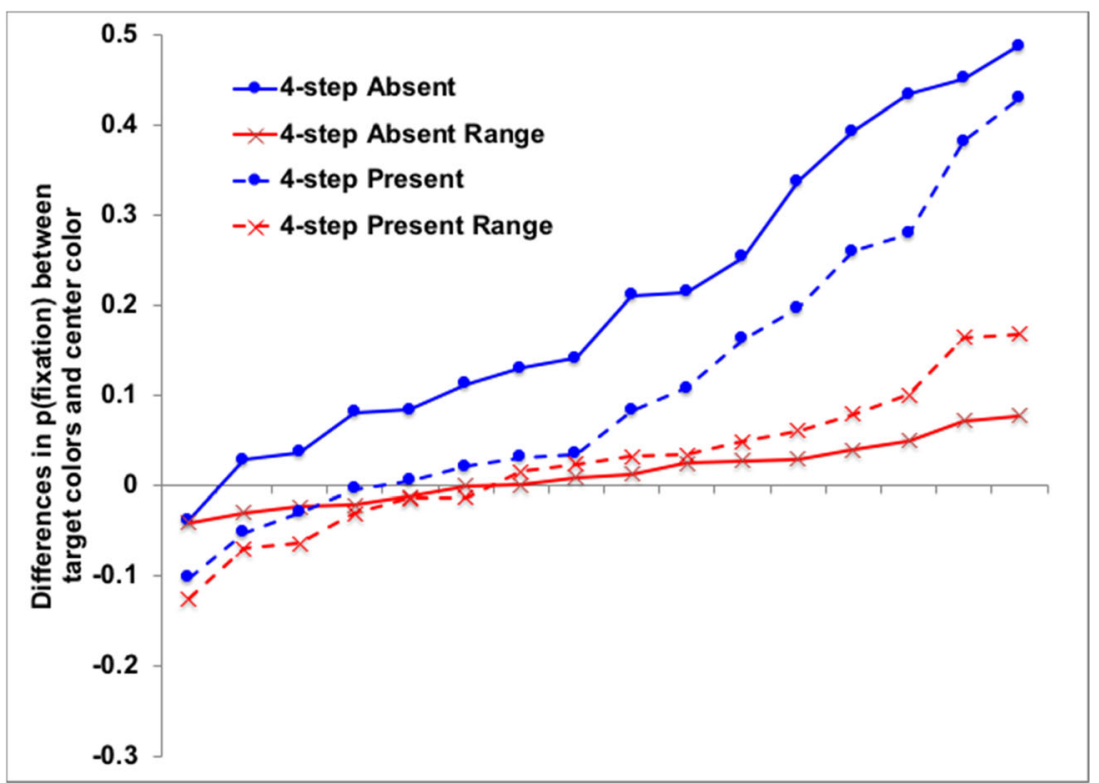

Fig. 13 The individual differences for the four-step range and four-step Target Sets in Experiment 5, ordered from lowest to highest differences independently for each condition. The $\mathrm{x}$-axis represents the individual subjects arranged in ascending order for each condition

they are part of the specified target color range. Subjects searched through the exact same stimulus arrays as the discrete four-step Target Set in Stroud et al. (2012), but in the preview before each trial, the target was specified as a continuous range of colors to search for instead of two separate objects. To provide the best comparison to Stroud et al. (2012), the target colors only appeared on the outer ends of the range of the rainbow stimulus. There were no differences in accuracy between the two Target Sets. There were differences in response time, and the fixation probabilities revealed just how strongly search was affected when subjects were given the target range (see Table 1).

Overall, subjects given the four-step rainbow stimulus fixated significantly more colors, indicating a reduction in color selectivity. The interesting finding was how the rainbow target specification affected subjects' use of color during search. When the target was present, subjects did in fact fixate the intervening colors at a higher frequency than when the target was specified as two separate objects, which is perhaps unsurprising given they were informed that these colors could be targets. However, and more importantly, the fixation probabilities for the two conditions also differed for colors least similar to the targets, and this difference was greater than for the intervening colors. It is surprising that the two different conditions would differ so dramatically in fixations to these leastsimilar colors, because subjects received similar information about these colors in the two conditions: both types of cues informed subjects that these colors could never be associated with targets. Nevertheless, when the target was specified as a range, subjects fixated all colors at a fairly high frequency when the target was absent, indicating a reduction in color selectivity for that condition.
These results suggest, as in Experiment 1, that search may be guided to some extent by the target colors, but if the target is not located, color guidance becomes less selective, allowing more fixations to colors that are not that similar to the target colors. It is possible that subjects are more resistant to searching for multiple targets within a range of colors rather than for two discrete targets, and that this resistance led subjects to change strategy; for example, to use the T-shape to identify the target. A shift from color to shape is surprising, given that the presentation of the target colors in Experiment 5 was designed to encourage subjects to think of the target colors as a unified range, and that Experiments 1-3 show that subjects can search effectively for a set of colors. Nonetheless, the fixation data are consistent with a shift to shape.

The individual differences were also consistent with the notion that search may be guided to some extent by multiple target colors. Figure 13 shows that the proportion of subjects with difference scores close to 0 (i.e., who fixated the color falling between the two targets as often as the targets) is greater for the four-step range condition than for the original fourstep discrete condition. However, these data may be misleading because subjects presented with the range fixated nearly every color at a greater frequency, and not just the intervening colors.

Subjects had difficulty utilizing the presented range target definition to guide search effectively. The target-color rainbow stimulus did cause a decrease in selectivity for the intervening colors, but it also created a much larger cost for objects dissimilar to either target color. Thus, it appears that for target colors that are separated by four steps in color space, subjects are not representing the set or range of colors to guide search in the current experiment, despite evidence from Experiments 
1-3 that subjects can use a set of colors to guide search. The next experiment therefore addresses whether a template range can be interpreted and used for two similar colored targets if there are fewer intervening distractor colors included.

\section{Experiment 6}

The results of Experiment 5 revealed that when subjects saw a depiction of a color range that included two target colors that were four steps apart on our ring of 16 colors (Fig. 1), they did not effectively guide search by color. Some small degree of color selectivity was maintained, although the effect of Color Step, as an indication of color guidance, was greatly reduced compared to conditions in which targets were presented as two or four discrete objects (see Table 1). The purpose of Experiment 6 is to examine if subjects will effectively search for a range of colors when the separation in color space between the two targets is reduced to just two steps. Stroud et al. (2012) found that all subjects in the two-step fixated the intervening color as often as they fixated the target colors, consistent with a combined representation for the two targets that included the intervening color. Their results also showed a cost directly related to the similarity between the search targets, with decreased color guidance associated with the decrease in similarity between the target colors. The current experiment aimed to investigate if this split-target cost may also be preventing the use of a target range. Subjects were presented with a target range that spanned only two-color steps (containing one intervening distractor color). As in Experiment 5, they searched for either of two targets on each trial.

\section{Method}

\section{Subjects}

Sixteen additional University of Massachusetts students, recruited from the same subject pool as the previous experiment, took part in this experiment (mean age $=19.4$, years $S D=0.9$ ).

\section{Design}

The data from the two-step Target set from Stroud et al. (2012), which used discrete target previews, were used to compare with the current experiment. The resulting design was a 2 (Target Set: two-step vs. two-step range) $\times 2$ (Target Presence: absent vs. present $) \times 9$ (Color Step) mixed factorial design with Target Set as the only between-subjects factor. The factor of Color step consisted of one level for the target colors, one level for the non-target color between the two target colors in color space, and seven for the non-target colors outside the two target colors.

\section{Apparatus and stimuli}

The same equipment and settings from the previous experiments were used for the current experiment. Subjects viewed exactly the same stimuli as in the discrete two-step Target Set from Stroud et al. (2012). The only difference was that in the preview before each trial, the targets were specified by a rainbow stimulus similar to Fig. 10, but only spanning three color steps (two target colors and one intervening color).

\section{Procedure}

The procedure was identical to Experiment 5.

\section{Results and discussion}

Firstly, the two-step range Target Set was compared with the two-step Target Set from Stroud et al. (2012) to evaluate the same predictions regarding representation of the targets as two separate objects versus one unitary range. Planned comparisons were conducted on fixation rates for the single intervening color between the two Target Sets. Secondly, the two-step range and the four-step range Target Sets were compared to determine how the range representation was influenced by the separation between the two target colors in color space. Finally, individual differences were examined.

\section{Two-step range versus two-step targets}

There was no reliable difference between the two-step and the two-step range Target Sets for both accuracy and response times (both $p$ 's $>.20$ ). Subjects responded faster and less accurately when the target was present (both $p$ 's $<.001$ ), and the Target Set $\times$ Target Presence interaction failed to reach significance for both measures (both $p$ 's $>.25$ ). See Fig. 4 for error rates and response time data.

Subjects fixated more objects on target-absent trials compared to target-present trials, $F(1,30)=234.38, p<.001$, and more objects similar to the targets (effect of Color Step: $F(8,240)=122.87, p<.001)$. Subjects given the range target specification fixated significantly more objects than those shown two separate target objects, both when the target was present, $F(1,30)=4.64, p=.039$, and when it was absent, $F(1,30)=1.31, p=.034$ (see Fig. 14). The only significant interaction was between Target Presence and Color Step, $F(8,240)=27.71, p<.001$, replicating the finding that subjects are more color selective when the target is present. The three-way interaction between Target Set, Target Presence and Color Step $(p=.250)$ and the two-way interaction between Target Set and Color Step $(p=.271)$ both failed to reach significance, which suggests that color was not used differently between the discrete and range Target Sets. Fixations to the intervening color did not significantly differ between the two 

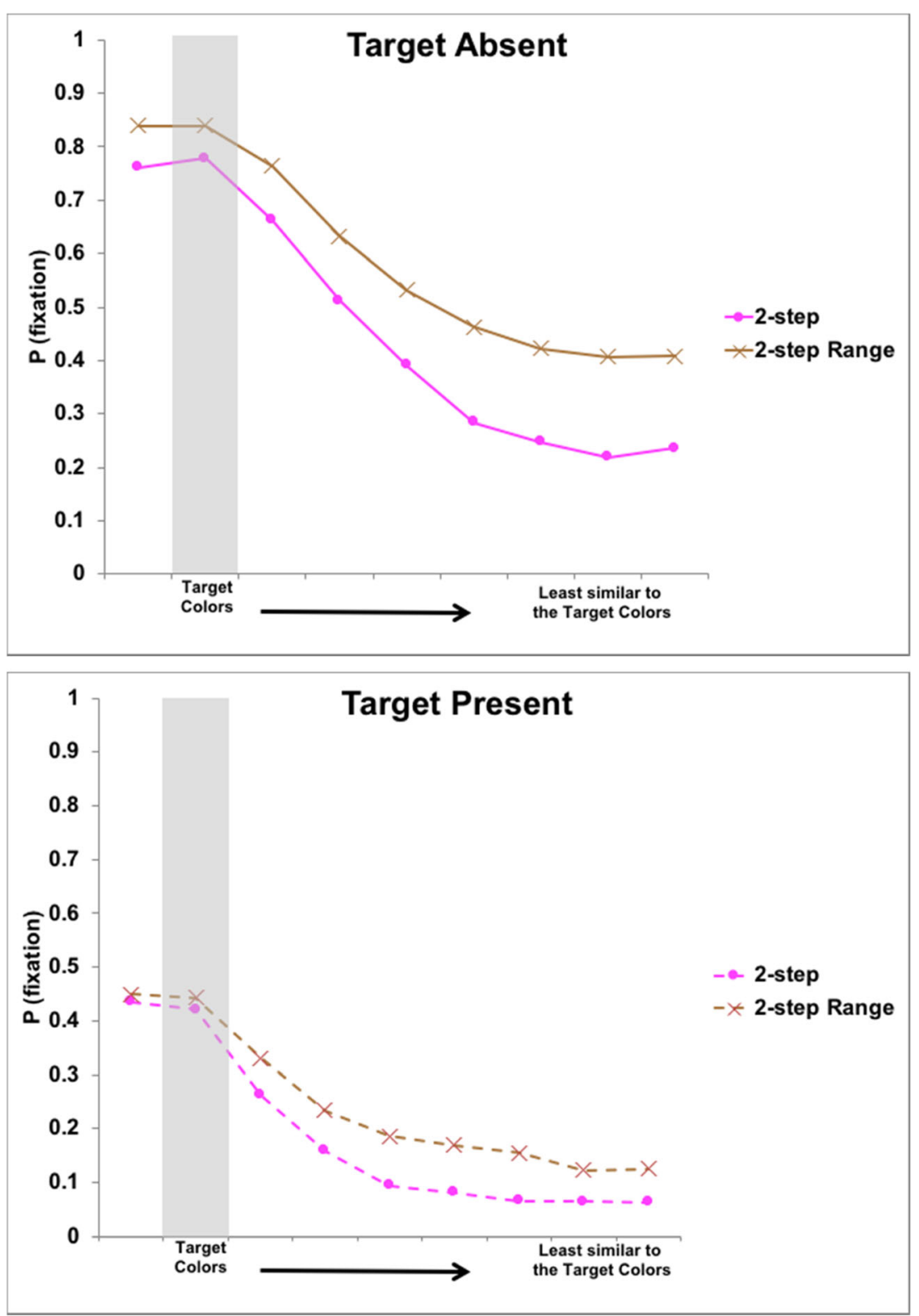

Fig. 14 The probability of fixation for the two-step and two-step range Target Sets in Experiment 6 for both target absent (top panel) and present (bottom panel) trials. The gray bar indicates the target colors in the two-

Target Sets for both target-absent $(p=.784)$ and target-present $(p=.144)$ trials.

\section{Two-step range versus four-step range}

The four-step range and two-step range Target Sets were compared to investigate the effect of the increased number of colors represented in the range on the objects fixated. Figure 15 shows that the four-step range was more difficult to maintain compared to the two-step range. Similar to the comparison between the two-step and four-step Target Sets (Stroud et al., 2012), color guidance was dramatically reduced as the number of intervening colors increased. Color step condition, which correspond to the colors at the two ends of the target-color rainbow stimulus shown to the subjects in the two-step range condition

Step once again significantly interacted with Target Set, $F(7,210)=11.39, p<.001$, and Target Presence, $F(7,210)$ $=7.23, p<.001$. Finally, the range stimulus had varying effects on search depending on the presence or absence of the target. When the target was present, color selectivity was somewhat preserved for both Target Sets, although to different extents. However, when the target was absent, subjects given the four-step rainbow stimulus fixated almost every color with the same frequency, while color selectivity was relatively high with the two-step rainbow. This conclusion was supported by a significant three-way interaction between Target Set, Target Presence and Color Step, $F(7,210)=7.32, p<.001$. 

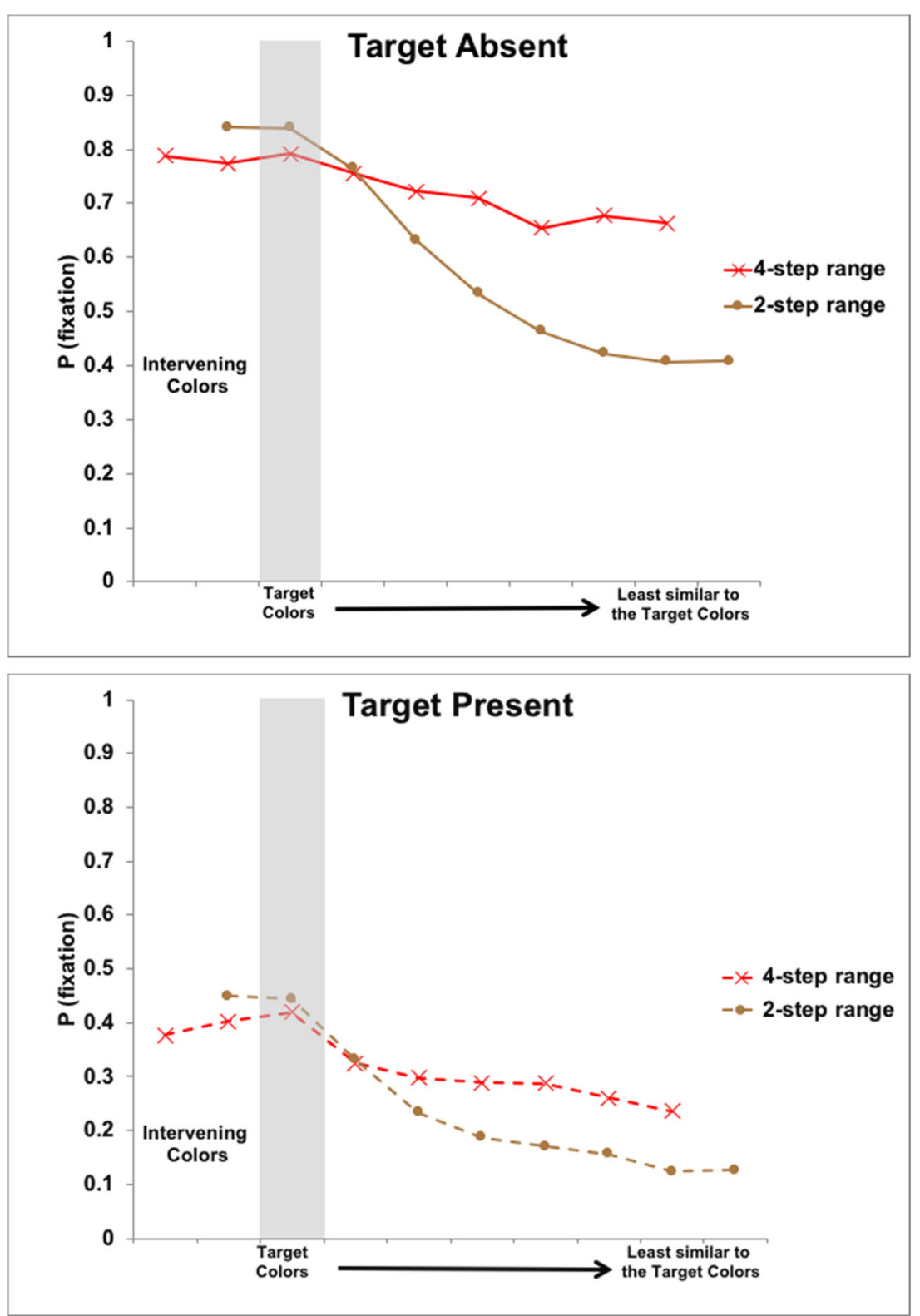

Fig. 15 The probability of fixation for the two-step range (Experiment 6) and four-step range (Experiment 5) Target Sets for both target absent (top panel) and present (bottom panel) trials. The gray bar indicates the colors at the two ends of the target color range shown to the subjects

\section{Individual differences}

Individual differences were evaluated as for the four-step Target Sets (Experiment 5) to determine whether subjects were maintaining two separate target representations or a unitary template range. Figure 16 presents these results for both the two-step and two-step range Target Sets. For the discrete Target Set (as reported in Stroud et al., 2012), no subjects fixated the intervening color at a significantly different rate than the target-color distractors, either in the target-present or the target-absent trials. This pattern also held for the range Target Set on target-present trials, while, on target-absent trials, two subjects of 16 fixated target-color distractors significantly more often than the intervening distractor color. The results demonstrate that in both conditions (discrete and range), the majority of subjects fixated the single, intervening color at about the same frequency as the target colors.

\section{Summary}

The results of the current experiment reveal a pattern of fixations for targets separated by two steps that may be consistent with a range representation. It is important to note that the twostep Target Set from Stroud et al. (2012) produced a similar pattern. However, the discrete representation model in Stroud et al. (2012) demonstrated that a two-representation account could produce these data, despite the lack of dip in the fixation rate for the intervening color, if we assume that the distractor color between the two target colors receives attentional 


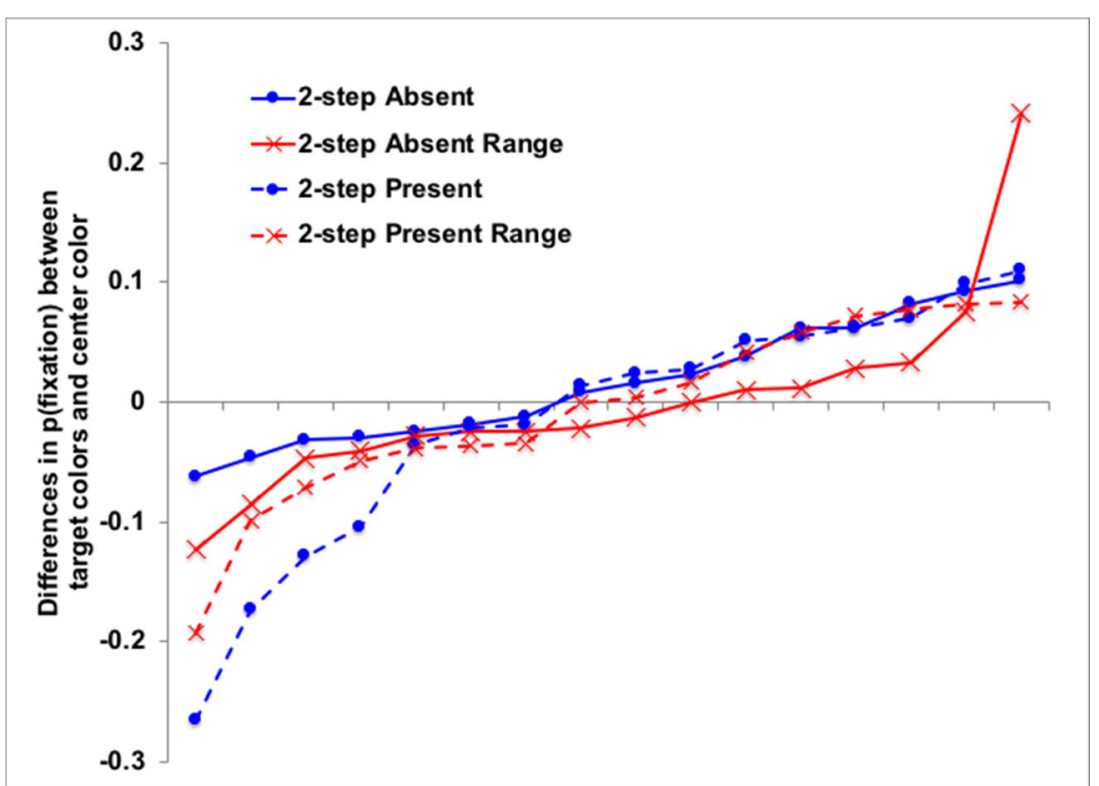

Fig. 16 The individual differences for the two-step and two-step range Target Sets in Experiment 6

activation from both targets. When there is only one color between the two target colors, the predictions from the discrete representation model are similar to what would be expected from a range representation (see Stroud et al., 2012 for details).

The results from Experiment 5 suggested that subjects might be completely unable to use a continuously changing color stimulus resembling a segment from a rainbow to construct an effective search target representation. Although it is unclear whether the target colors guiding search in Experiment 6 are mentally represented as a pair of discrete colors or as a single continuous range, the search performance shows that the initial depiction of the target colors as a continuous range at the beginning of each trial can be used relatively well to build an effective target representation in some circumstances.

The difference in selectivity between Experiments 5 and 6 could have emerged because the color information provided to participants in Experiment 5 is less informative than it is in Experiment 6, given that the target preview captures more stimulus colors that are not actually targets. There may be other manipulations that will allow subjects to create an effective target representation from such a stimulus; for instance, if all the possible colors in the depicted color range actually appeared as targets, we might expect fixation patterns similar to those in Experiments 1 and 2. Whether search with these depictions of the target set can be improved or not, these results show that translating these continuous stimuli into an effective target representation is not as straightforward as we expected.

\section{Experiment 7}

Experiments 5 and 6 and the experiments from Stroud et al. (2012) varied the information given to subjects about possible targets in an attempt to influence the representations utilized to guide fixations during search, but did not change the targets or distractors. Rather than manipulating the information given before the stimuli appeared, the current experiment tested the limits of selectivity by manipulating the distractors that appeared across trials. In Experiment 7, we presented subjects with the same target information as used in Stroud et al. (2012), i.e., two separate target colors, but removed distractors of colors that fell between the two targets for the first half of the experiment.

Previous research has shown that the saccadic system is quite flexible in tailoring the pattern of fixations according to characteristics of the stimuli (Pomplun, Reingold, \& Shen, 2001, 2003; Shen, Reingold, \& Pomplun, 2000, 2003; Williams, \& Reingold, 2001). Given this flexibility, the goal of the following experiment is to investigate how the guiding representations are shaped by the absence of distractor colors that fall between the two target colors in the color stimulus space. In this situation, it may be more efficient to combine the target colors into a single representation: the intervening colors could be contained within the representation, but would not be a disadvantage given that they do not appear as distractors.

In the four-step condition in Stroud et al. (2012), when the targets are separated by four color steps, search seemed to be generally guided by two separate representations for each target, as evidenced by an ability to limit fixations to the intervening colors. In Experiment 5, prompting subjects with a rainbow representation of the target colors produced reduced guidance. In both of these conditions, distractors could appear in colors that fell between the two target colors in color space. Perhaps a unified range representation is more likely to be used in dual-target search when there are no intervening distractor colors included as part of that representation. Moore and Weissman (2010) reported that exposure to distractors that share features of the target may bias 
the focus of attention. If subjects have the flexibility to represent the targets as two separate colors or as a range of values, they may be more likely to switch to representing colors separately if they encounter distractors with colors that fall in between the two targets (in color space), thereby making search more effective. If intervening colors influence the representation(s), then eliminating exposure to intervening distractors may allow a range representation of the target colors to form. Excluding the intervening distractors makes the search task more like D'Zmura's (1991) dual-target searches in which target colors were linearly separable from the distractor colors, and which led to efficient search.

The current experiment aims to test if a single range representation might be conjured if subjects do not encounter any distractors with these intervening values for the first half of the experiment. Such a range representation could encompass both target colors along with the intervening colors that never appear during the first half, and would lead to efficient search. During the second half of the experiment, intervening colors will be presented (as distractors) as in the four-step Target Set from Stroud et al. (2012) in order to test whether the target representation is directing fixations to those colors. If a single range target representation is created and maintained from the first half, then in the second half the intervening colors should be fixated at rates similar to the targets.

Specifically, this question was investigated in the current experiment by comparing the fixation rates for the intervening colors between the second half of the current experiment and the discrete four-step Target Set from Stroud et al. (2012). Only the four-step Target Set was used in the current experiment because it is the condition from the previous experiments in which the targets are least similar to one another, meaning that the targets have more intervening colors between them, and thus it is the condition in which it is easiest to see differences between a range representation and a representation of two discrete colors. If the proportion of fixations to intervening distractors in the second half of the current experiment is significantly higher than in the latter half of the four-step Target Set, it will be evidence that a range representation has been formed or has been strengthened in response to the distribution of the stimuli encountered.

In summary, the current experiment is aimed at exploring the following question: If there are no distractors with colors between the two target colors, will subjects be more likely to include those colors in a unified target representation?

\section{Method}

\section{Subjects}

Sixteen additional subjects from the same pool as the previous experiments participated in Experiment 7 (mean age $=19.6$ years, $S D=1.4$ ).

\section{Design}

There were three factors in the current experiment. First, the Distractor Set factor consists of the four-step condition from Stroud et al. (2012), and the new condition that has no intervening distractor colors for the first half of the trials. The search target colors were the same for both levels of this factor and Distractor Set refers to the presence or absence of intervening colors during the $1^{\text {st }}$ half of the experiment. The resulting design was a 2 (Distractor Set: intervening vs. nointervening) $\times 2$ (Target Presence: absent vs. present $) \times 7$ (Color Step: Target -6 steps) mixed ANOVA. The nontarget colors that were between the two target colors in color space were not included in the analysis.

\section{Apparatus}

The same equipment and settings as the previous experiments were utilized for the current experiment.

\section{Stimuli}

The stimuli and task were the same as Stroud et al. (2012): at the beginning of each trial, subjects were presented with two Ts to search for, one in each of the two possible target colors. The main manipulation was to eliminate intervening distractor colors during the first half of the experiment. The first 128 trials only contained objects drawn from 13 out of the 16 possible colors from the color wheel: the two target colors and the remaining 11 distractor colors "outside" the targets. Because these trials do not contain any intervening colors, the total pool of possible objects to create the stimuli was reduced, and the additional distractors were evenly distributed across the remaining 13 distractor colors. This elimination of intervening-colored distractors caused the probability of a target-colored item being a target to be slightly lower in the first half $(25 \%)$ than the second half $(30 \%)$.

Subjects were not informed of the composition of the stimuli. To allow a direct comparison with the four-step condition from Stroud et al., the second half of this experiment contained the exact same trials, including the intervening colors. The purpose of this condition was to test whether repeated trials with no intervening colors affected subjects' selectivity on subsequent trials. That is, after not being exposed to the intervening colors, are those feature values effectively "turned off" or "enhanced" in the attentional system?

\section{Procedure}

The procedure was identical to Experiments 1-4, except that the target preview comprised two targets. 


\section{Results and discussion}

The results of four-step condition from Stroud et al. (2012) were included in the data analysis, reflecting the condition in which the intervening colors appeared as frequently as the colors outside the target colors in the stimulus color space. This four-step condition contains intervening colors on $86.5 \%$ of the trials $(S D=1.41 \%)$. For the purposes of the current experiment, the condition matching Stroud et al. will be referred to as "intervening" and the new condition will be labeled "no-intervening" to focus on the most important aspect that is being compared.

Error rates and response times were submitted to a 2 (Target Presence: absent vs. present) $\times 2$ (Distractor Set: intervening vs. no-intervening) mixed analysis of variance, with Distractor Set as a between-subjects factor. In order to compare the effects of exposure to the intervening colors, the first and second halves of the experiments were analyzed separately.

Consistent with all previous analyses, subjects committed more errors, but responded faster, when the target was present (ps< .001) (see Fig. 11). There was no significant difference for response times between the two Distractor Sets for the first half of the trials. However, subjects were slightly more accurate with the no-intervening (mean error rate $=4.5 \%$ ) than intervening (7.1\%) Distractor Set, $F(1,30)=5.25, p=.029$, especially when the target was present, resulting in a significant Distractor Set $\times$ Target Presence interaction $F(1,30)=$ $1.30, p=.047$. This may reflect the fact that there were fewer distractors that were very similar to the target in color in the no-intervening condition.

For the second half of the experiment, error rates and response times did not significantly differ between the two Distractor Sets. There was a significant Distractor Set $\times$ Target Presence interaction, $F(1,30)=5.54, p=.025$, for response times only, as a result of faster responses for the no-intervening than the intervening Distractor Set when the target was present.

To evaluate the hypotheses regarding the influence the intervening colors had on selectivity, the fixation probabilities were analyzed in a 2 (Distractor Set $) \times 2($ Target Presence $) \times 5$ (Color Step) mixed ANOVA. The fixation probabilities are presented in Fig. 17. As with the measures above, the two halves of the experiment were compared separately. Only the colors common across the two Distractor Sets were included in each analysis. The results showed the same consistent finding that subjects fixated significantly more distractors similar to the targets (compared with dissimilar distractors); also they fixated more objects overall when the target was absent versus present $(p s<.001)$. Color Step and Target Presence significantly interacted both for the first half, $F(6,180)=$ $10.41, p<.001$, and the second half, $F(8,240)=21.86, p<$ .01 , which, again is a reflection of an increased fixation frequency on distractors that are dissimilar to target colors during target-absent trials.
There were no significant main effects or interactions involving Distractor Set for either the first half (all $p s>.91$ ) or the second half (all $p s>.45$ ). The bottom-left panel of Fig. 17 shows that fewer intervening colors were fixated in the second half for the intervening Distractor Set (compared with nointervening) when the target was present, but the comparison did not reach significance, $F(1,30)=1.46, p=.236$.

These results suggest that the intervening colors have, at most, a minimal influence on shaping the guiding representation (see Table 1). The error rates and response time data revealed limited but significant differences in performance for subjects exposed to the intervening colors. These effects may reflect differences in decision processes after visual items are selected. There was no converging support of these differences in the eye movement data, and thus there is no evidence that attentional guidance varied across conditions. It is true that fixation rates are numerically lower for the intervening compared to the no-intervening condition in the second half of trials. This difference hints that there might be some advantage for viewing intervening colors on previous trials, but it does not reach statistical significance. The low fixation rates for the intervening colors for the second half trials show that search was guided by two separate target color representations in both conditions, and the similarity in fixation patterns across both conditions suggests that subjects consistently used the same two target color representations in both, including the first half trials in the no-intervening condition. Even though a single range representation might have worked well with no intervening distractor colors, there is no evidence that subjects got in the habit of using one.

This result, combined with the findings from Experiments 5 and 6 , illustrate that it is not easy to encourage subjects to guide search with a single unified template that represents a range of colors. This does not mean that such a range template is never used, and the search guidance for a set of eight different colors in Experiment 3 may very well have been accomplished with such a range template. However, further experiments will be necessary to determine whether search can actually be guided in this way.

\section{General discussion}

The purpose of the current set of experiments was to investigate whether search can be guided effectively by a large set of colors that are taken from a single region in the color space from which all non-target colors have been excluded. Previous research involving search for multiple targets has shown that in many circumstances, two targets are represented as discrete templates (Barrett \& Zobay, 2014; Beck \& Hollingworth, 2017; Beck, Hollingworth, \& Luck, 2012; Grubert \& Eimer, 2015, 2016; Irons, Folk, \& Remington, 2012; Stroud et al., 2012). However, little work has examined whether subjects 


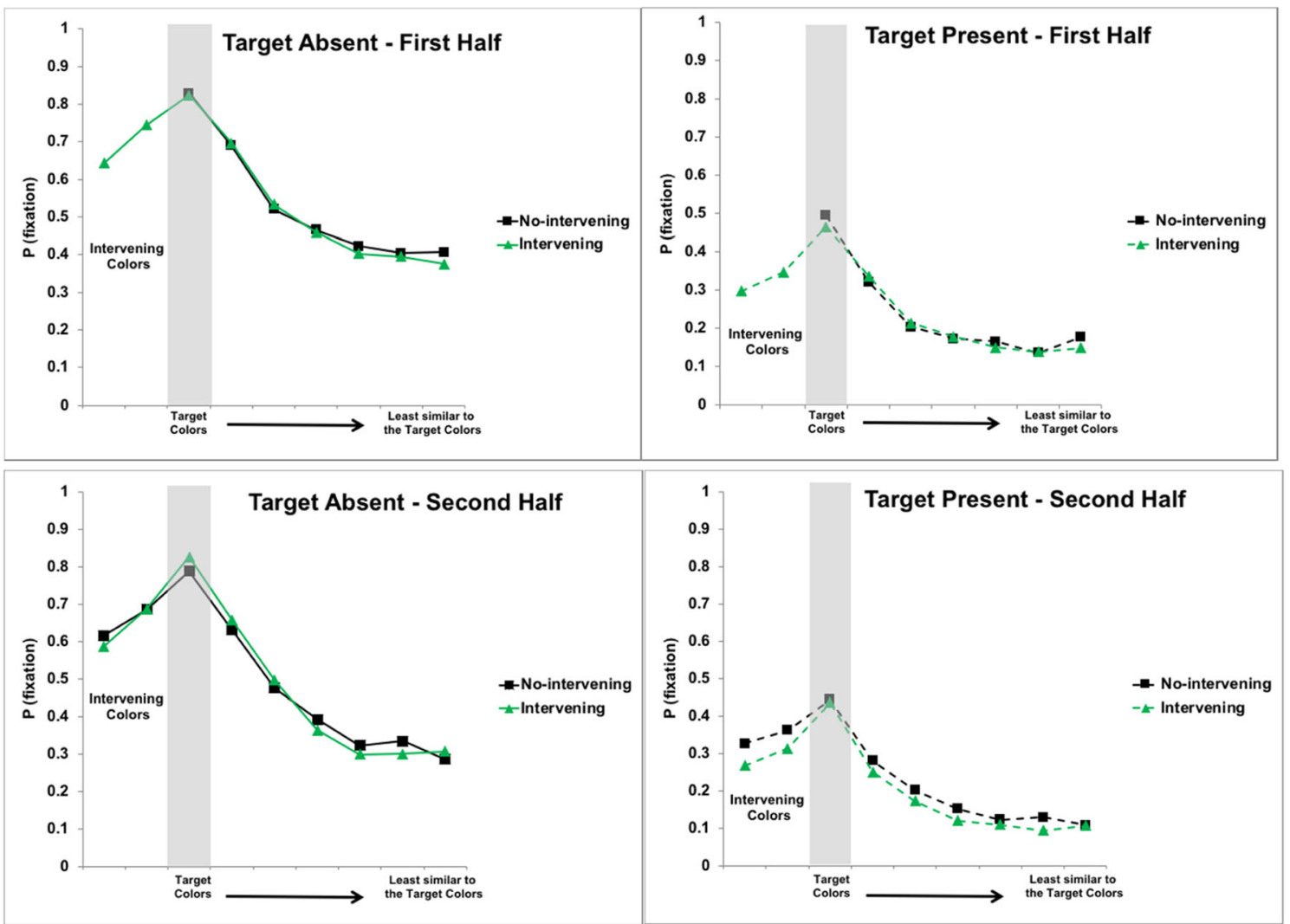

Fig. 17 The probability of fixation for the Intervening and No-intervening Distractor Sets in Experiment 7 . The top panel represents the first half of each experiment and the bottom panel represents the second half

can search effectively for a set of multiple colors that are similar to one another such that they can be represented by a single region of color space that does not also include distractor colors. The current approach involved manipulating the relative bottom-up information (relationship between color and target presence) and top-down guidance (specification of the target colors) in different combinations to reveal the dynamics of this complex representation.

The first four experiments manipulated the informativeness of the target colors while specifying those colors with discrete objects. Experiments 1 and 2 presented the target colors as four discrete color patches. The results demonstrated that when subjects were provided with four colors to search for that covered one-quarter of the possible hues, they were generally able to guide eye movements to those colors, regardless of how likely target colors were to be target objects.

Experiment 3 tested whether this apparent range pattern seen with four discrete target colors could be extended to eight target colors, which make up half of the available hues. Target-color probability was high in this search: each appearance of a target color was more likely to be a target than a distractor. The fixation rates showed that search was guided by all eight colors. Experiment 4 employed a very low target probability in order to test whether this probability ever affects guidance for multiple colors, given that it made no difference in Experiments 1 and 2 (four-color HTP and four-color LTP). The results showed very little color selectivity. In Experiments 3 and 4 , as in all of the other experiments presented here, each subject searched for a different set of target colors, and there may be differences in the ability to guide search across these different target sets, but the same target sets were used for both Experiments 3 and 4, so the differences in guidance between these experiments cannot be attributed to differences in target sets. Combined, Experiments 3 and 4 show that subjects construct a target representation that includes a set of eight hues, but only if the color information is informative about target presence. The lack of guidance in Experiment 4 also demonstrates that although attentional mechanisms are available to make search more efficient, they are not always used.

With the possibility of search guidance by a large set of colors established in Experiments 1-3, the final three experiments explored whether search for a similar range of colors could be encouraged in the dual-target search tasks used by Stroud et al. (2012), in which target colors are separated by distractor colors in stimulus color space. Experiments 5 and 6 provided a rainbow-like color preview specifying a range that spanned either four color steps (Experiment 5) or two color steps (Experiment 6). Instead of producing effective search templates of the range of colors, these target color depictions led to less efficient search compared to previews consisting of discrete 
objects, as shown by reduced color selectivity for targets separated by four color steps and increased fixations overall for targets separated by two steps. This poor guidance might have been attributed to the difficulty of holding three or five possible target colors in visual working memory, but Experiments 1-4 demonstrate that working memory can hold the required amount of information and make it available for guiding eye movements. Perhaps working memory can represent a set of discrete colors, but cannot represent the sort of continuous range that is suggested by the rainbow stimulus. When given the visual depiction of a color range specifying target colors, subjects might try to encode a few specific colors from that range that are easily identifiable or easy to encode in memory. The different target ranges may vary in the number of easily encodable colors that they have. Once a set of colors is encoded in a target template, subjects may then attempt to guide their eyes towards the colors in the template and to similar colors.

Another possible explanation for the poor search performance is that the broad range of colors specified in the preview contained some colors (in the middle) that were never actual target colors, and thus may have created a level of interference. Experiment 7 assessed the flexibility of the target representation by manipulating the distribution of distractor colors that the observers encountered. Specifically, does exposure to the distractor colors in between the two target colors (in color space) shape the guiding template or templates? If subjects did not view distractors with any of these intervening colors as they became familiar with the search task, then perhaps they would represent the two target colors as a range of values including the targets and the colors in between, because there would be no advantage to excluding the intervening colors. If this was the case, then fixations to the intervening colors should occur at the same frequency as fixations to the targets for the second half of the experiment. The results did not support this conclusion, and revealed that exposure to the intervening colors had little to no effect on shaping the target template.

In summary, there are four main conclusions to draw from these experiments. The first is about subjects' ability to search for a large set of colors. Earlier results had suggested that a range template for attentional guidance was simply not possible. Stroud et al. (2012) applied a simple, discrete representation model that provided evidence for separate representations for both similar and dissimilar colored targets in search for two objects. Similar accounts of multiple sources of attentional guidance have been provided by several other researchers (Barrett \& Zobay, 2014; Beck et al., 2012; Schwark, Dolgov, Sandry, \& Volkman, 2013). However, the new experiments presented here show that under the right circumstances, subjects will create a representation encompassing a set of similar colors and use it to guide eye movements.

The other three conclusions provide insight into what it takes to allow observers to construct an effective unified template in conditions that have previously led to separate target templates.
The second conclusion demonstrates that even though the rainbow-like stimulus used in Experiments 5 and 6 intuitively seems to be a very accessible way of illustrating the idea of a range that covers a region in color space, subjects were unable to translate it into an effective search template. Instead, for these search tasks, in which the targets and distractors are presented as separate objects each with its own single uniform color, subjects can more effectively interpret a target preview consisting of the same type of separate, uniformly colored objects.

The third conclusion is that constructing and maintaining a representation of a set of colors becomes more difficult as the size of the range increases (see effect of Color Step for fourcolor HTP vs. eight-color HTP in Table 1). It should be noted that none of the color-set searches in these experiments comes close to the efficiency of the searches for a single specific target in Stroud et al. (2012). As the size of the target set increases, more conditions have to be met before eye movements are guided by the target set, suggesting that broader templates are more difficult to create and to use. Experiment 6 shows some degree of color selectivity for a two-step range, which includes one-eighth of the hue dimension, even when the range is specified by a rainbow-like stimulus. In Experiment 2, search guidance is fairly effective for a fourcolor target set, which covers one quarter of the hue dimension. The same type of preview is also sufficient for creating an eight-color template when the target colors are reliable cues to target presence, presumably because this reliability warrants the effort in maintaining the color information.

That brings us to the fourth and final conclusion. Observers may be sensitive to the informativeness of the target preview, in terms of the frequency with which a specified target color actually signals the presence of a target, but this sensitivity may be limited to target sets that include a large number of target values. The effect of target color frequency is seen in the comparison of fixation rates from Experiments 3 and 4: both experiments require search for the same set of eight colors, but guidance is much less effective when many distractors appear with target colors. This sensitivity to the informativeness of target features may only arise with complex target feature sets such as the eight-color set in Experiments 3 and 4. More tests will be needed to know if search will be guided by smaller color sets when the target color probability is very low.

Thus, overall, observers do have the ability to represent and search for a large set of colors, but this increase in the number of target colors comes with a cost to selectivity.

These results lead to interesting questions for future studies. There is much still to be learned about why search guidance is so ineffective in some circumstances. Experiment 5 provides a particularly good example of a situation in which guidance is very poor even though all of the information is available to construct an effective representation of the target colors. This is one case in which subjects are clearly not using their attentional mechanisms as effectively as they could. The 
rainbow stimulus does include incorrect information about the target colors, given that the targets could only appear in the end colors, so it could be argued that search was disrupted by including non-target colors in the target information. However, limiting search only to that portion of color space presented in the rainbow stimulus would have made search and guidance more effective than observed.

One important aspect of all of these experiments, and also of the experiments in Stroud et al. (2012), is that both color and shape information are relevant to finding and identifying a target. When it comes to guiding attention and eye movements, it is much easier to find a specific color among a multicolor set of distractors than to find a rotated $\mathrm{T}$ among rotated L's. Nonetheless, because the target is always a $\mathrm{T}$ and the distractors are all Ls in these experiments, it is possible to find the target purely by shape while ignoring color. Thus, the results of Experiments 1-3 demonstrate not only that search guidance by a set of colors is possible, but also that under the right circumstances, subjects will elect to engage this multicolor guidance rather than falling back on detecting targets by their shape alone.

The ability to detect targets without color guidance in these searches means that subjects may fall back on detecting the target shape in some situations, and if they do, these results may not reflect the best possible color guidance. We have tested this possibility in a separate series of experiments in which the target can only be identified by color. In these circumstances, the eyes are guided fairly effectively by color, even in dual-target search (Cave, Menneer, Kaplan, Stroud \& Donnelly, in preparation). These experiments provide additional evidence that we have very effective mechanisms for controlling attention and eye movements, but, as shown by the current experiments, we do not always use them effectively.

These experiments also raise questions about the role of working memory in guiding search. When subjects guide search for multiple colors, are they storing the target representation in the same memory store that is used for other visual tasks? A number of recent studies have explored the interaction between visual working memory and attentional control. Based on their measurements of the CDA (contralateral-delay activity; a measurement of current object representation in working memory), Carlisle, Arita, Pardo, and Woodman (2011) concluded that when subjects search for the same targets across many trials, the search targets come to be represented independently of working memory. In addition, other recent evidence suggests that search may be guided by episodic longterm memory as opposed to simply visual working memory, given that search can be successful for large numbers of targets (Cunningham \& Wolfe, 2014; Giammarco, Paoletti, Guild, \& Al-Aidroos, 2016; Wolfe, 2012; Wolfe et al., 2016). In the current set of experiments, the search targets are simple and consistent across every trial. Therefore, long-term memory representations may be formed, freeing up attentional resources for search. These findings would suggest that working memory is not critical in these search tasks, in which the target colors are consistent for each subject. Other experiments, however, show a strong link between working memory and search performance on these searches. Menneer, Cave, Kaplan, Stroud, and Donnelly (submitted) have coupled a singletarget version of the specific visual search task used here with different visual working memory tasks. The results suggest a complex relationship between the target representation and visual working memory: if subjects are holding a color in visual working memory, their guidance of eye movements by color is much less effective. However, there is not much confusion between the memory color and the search target color; subjects do a fairly good job of keeping the two separate. Thus, it appears that maintaining the search target representation draws on some of the same resources as other visual memory tasks, but the representations are fairly well separated.

The question of working memory storage is especially important in Experiment 3, in which subjects guide search to a set of eight different target colors. Perhaps, as Carlisle et al. suggest, the target colors are transferred to long-term memory. Indeed, an exploratory examination of our dual-target search data split by quarters of the experiment (each containing 64 trials) showed an improvement in selectivity across trials, with peak selectivity being achieved half way through the trials. Such improvement could be attributed to task learning or to targets being supported by long-term memory. If instead they are held in working memory, it is not clear whether they are represented as eight separate items, or as a single range representation that covers all eight colors, or as something in between, such as a small set of small-range representations. It would be instructive to test whether subjects are able to search for a large set of colors that are not adjacent on the ring of possible colors, but come from a part of color space that, if selected as a contiguous region, would also contain distractor colors. Can they guide their eyes to the target colors without also selecting distractor colors between the target colors in color space? Answering this question may be hard, though, because Stroud et al. (2012) have shown that a single template for a range of colors can produce fixation patterns similar to a combination of templates for individual colors.

Acknowledgements Thanks to Keith Rayner, Matthew Davidson, Donald Fisher, and Junha Chang for valuable guidance; to Erica Kreindel, Ross Krebs, Shanila Sattar, Megan Curtis, and Jackie Cheng for help with testing subjects and analyzing the results; and to Olivia Downer and Ruja Kambli for suggestions on the manuscript. The authors would also like to thank Katherine Moore and Jeremy Wolfe for their helpful comments regarding the manuscript.

\section{References}

Barrett, D. J. K., \& Zobay, O. (2014). Attentional control via parallel target-templates in dual-target search. PLOS ONE, 9(1), e86848. 
Beck, V. M., \& Hollingworth, A. (2017). Competition in saccade target selection reveals attentional guidance by simultaneously active working memory representations. Journal of Experimental Psychology: Human Perception and Performance, 43(2), 225-230.

Beck, V. M., Hollingworth, A., \& Luck, S. J. (2012). Simultaneous control of attention by multiple working memory representations. Psychological Science, 23(8), 887-898. https://doi.org/10.1177/ 0956797612439068

Becker, S. I (2010). The role of target-distractor relationships in guiding attention and the eyes in visual search. Journal of Experimental Psychology: General, 139(2), 247-265. https://doi.org/10.1037/ a0018808

Becker, S. I., Folk C.L., \& Remington, R. W. (2013). Attentional capture does not depend on feature similarity, but on target-non-target relations. Psychological Science, 24(5), 634-647. https://doi.org/10. 1177/0956797612458528

Carlisle, N.B.,Arita, J. T., Pardo, D., \& Woodman, G. F. (2011). Attentional templates in visual working memory. The Journal of Neuroscience, 31, 9315-9322.

Cave, K. R., Menneer, T., Kaplan, E., Stroud, M. J., \& Donnelly, N. (In preparation). Effective Visual Search Guidance with Multiple Items in Working Memory: The Dual-Target Cost and the WorkingMemory Cost Can be Largely Avoided.

Cave, K. R., Menneer, T., Nomani, M.S., Stroud, M. J., \& Donnelly, N. (2018). Dual Target Search is Neither Purely Simultaneous nor Purely Successive, Quarterly Journal of Experimental Psychology, 71, 169-178.

Cunningham, C. A., \& Wolfe, J. M. (2014). The role of object categories in hybrid visual and memory search. J Exp Psychol Gen, 143(4), 1585-1599. https://doi.org/10.1037/a0036313

Desimone, R., \& Duncan, J. (1995). Neural mechanisms of selective visual attention. Annual Review of Neuroscience, 18, 193-222.

D'Zmura, M. (1991). Color in visual search. Vision Research, 31(6), 951966.

Folk, C. L., Remington, R. W., \& Johnston, J. C. (1992). Involuntary covert orienting is contingent on attentional control settings. Journal of Experimental Psychology: Human Perception \& Performance, 18(4), 1030-1044

Forder, L., He, X., \& Franklin, A. (2017). Colour categories are reflected in sensory stages of colour perception when stimulus issues are resolved. PLoS One, 12(5): e0178097. https://doi.org/10.1371/ journal.pone.0178097

Giammarco, M., Paoletti, A., Guild, E., \& Al-Aidroos, N. (2016). Attentional capture by items that match episodic long-term memory representations. Visual Cognition, 24(1), 78 - 101

Grubert, A., \& Eimer, M. (2015). Rapid parallel attentional target selection in single-color and multiple-color visual search. Journal of Experimental Psychology: Human Perception and Performance, 41(1), 86-101. https://doi.org/10.1037/xhp0000019

Grubert, A., \& Eimer, M. (2016). All Set, Indeed! N2pc Components Reveal Simultaneous Attentional Control Settings for Multiple Target Colors. Journal of Experimental Psychology: Human Perception and Performance https://doi. org/10.1037/xhp0000221

Hollingworth, A., \& Beck, V. M. (2016). Memory-based attentional capteru when multiple items are maintained in visual workingmemory. Journal of Experimental Psychology: Human Perception and Performance, 42(7), 991-997.

Hout, M. C., \& Goldinger, S. D. (2010). Learning in repeated visual search. Attention, Perception \& Psychophysics, 72, 1267-1282.

Hout, M. C., \& Goldinger, S. D. (2015). Target templates: The precision of mental representations affects attentional guidance and decision-making in visual search. Attention, Perception \& Psychophysics, 77, 128-149.

Houtkamp, R., \& Roelfsema, P. R. (2009). Matching of visual input to only one item at a time. Psychological Research, 73, 317-326.
Irons, J. L., Folk, C. L., \& Remington, R. W. (2012). All set! Evidence of simultaneous attentional control settings for multiple target colors. Journal Of Experimental Psychology: Human Perception And Performance, 38(3), 758-775. https://doi.org/10.1037/a0026578

Ishihara, S. (1917), Tests for colour-blindness Handaya, Tokyo, Hongo Harukicho

Luck, S. J., \& Vogel, E. K. (1997). The capacity of visual working memory for features and conjunctions. Nature, 390,279-281.

Menneer, T., Barrett, D. J. K., Phillips, L., Donnelly, N., Cave, K. R. (2004). Search efficiency for multiple targets. Cognitive Technology, 9, 22 - 25.

Menneer, T., Barrett, D. J. K., Phillips, L., Donnelly, N., \& Cave, K. R. (2007). Costs in searching for two targets: Dividing search across target types could improve airport security screening. Applied Cognitive Psychology, 21(7), 915-932.

Menneer, T., Cave, K.R., \& Donnelly, N. (2009). The cost of searching for multiple targets: The effects of practice and target similarity. Journal of Experimental Psychology: Applied, 15, 125-139.

Menneer, T., Cave, K.R., Kaplan, E., Stroud, M.J. \& Donnelly, N. (submitted) Working memory interactions play a fundamental role in the dual-target cost in visual search guidance.

Menneer, T., Donnelly, N., Godwin, H. J., \& Cave, K. R. (2010). High or low target prevalence increases the dual-target cost in visual search. Journal of Experimental Psychology: Applied, 16, 133-144.

Menneer, T., Stroud, M. J., Cave, K. R., Li, X., Godwin, H. J., Liversedge, S. P. \& Donnelly, N. (2012). Search for two categories of target produces fewer fixations to target-color items. Journal of Experimental Psychology: Applied. Vol 18(4), 404-418.

Moore, K. S., \& Weissman, D. H. (2010). Involuntary transfer of a topdown attentional set into the focus of attention: Evidence from a contingent attentional capture paradigm. Attention, Perception, \& Psychophysics, 72(6), 1495-1509. https://doi.org/10.3758/ APP.72.6.1495

Moore, K. S., \& Weissman, D. H. (2011). Set-specific capture can be reduced by pre-emptively occupying a limited-capacity focus of attention. Visual Cognition, 19(4), 417-444.

Moore, K. S., \& Weissman, D. H. (2014). A bottleneck model of setspecific capture. PLOS ONE, 9(2), e88313.

Navalpakkam, V., \& Itti, L. (2007). Search goal tunes visual features optimally. Neuron, 53(4), 605-617. https://doi.org/10.1016/J. Neuron.2007.01.018

Neitz, J., \& Jacobs, G. H. (1986). Polymorphism of the long-wavelength cone in normal human color vision. Nature. 323 (6089): 623-5. https://doi.org/10.1038/323623a0.

Olivers, C. L., Peters, J., Houtkamp, R., \& Roelfsema, P. R. (2011). Different states in visual working memory: When it guides attention and when it does not. Trends In Cognitive Sciences, 15(7), 327-334.

Pomplun, M., Reingold, E. M., \& Shen, J. (2001). Investigating the visual span in comparative search: The effects of task difficulty and divided attention. Cognition, 81(2), B57-b67.

Pomplun, M., Reingold, E. M., \& Shen, J. (2003). Area activation: a computational model of saccadic selectivity in visual search. Cognitive Science, 27(2), 299-312. https://doi.org/10.1016/S03640213(03)00003-X

Schönhammer, J. G., Becker, S. I., \& Kerzel, D. (2017a). Which kind of attention is captured by cues with the relative target color? Visual Cognition, 25(7-8), 703-714. https://doi.org/10.1080/13506285. 2017.1323811

Schönhammer, J. G., Grubert, A., Kerzel, D. \& Becker, S. I. (2017b). Attentional guidance by relative features: Behavioral and electrophysiological evidence. Psychophysiology, 53, 1074-1083.

Schwark, J. D., Dolgov, I., Sandry, J., \& Volkman, C. B. (2013). Simultaneous attentional guidance by working-memory and selection history reveals two distinct sources of attention. Acta Psychologica,144(2), 269-278. https://doi.org/10.1016/j.actpsy. 2013.06.017 
Shen, J., Reingold, E. M., \& Pomplun, M. (2000). Distractor ratio influences patterns of eye movements during visual search. Perception, 29(2), 241-250.

Shen, J., Reingold, E. M., \& Pomplun, M. (2003). Guidance of eye movements during conjunctive visual search: The distractor-ratio effect. Canadian Journal of Experimental Psychology/Revue Canadienne De Psychologie Expérimentale, 57(2), 76-96.

Sobel, K. V., Puri, A. M., \& Hogan, J. (2015). Target grouping in visual search for multiple targets. Attention, Perception and Psychophysics, 77, 67-77.

Stroud, M. J., Menneer, T., Cave, K. R., \& Donnelly, N. (2012). Using the dual-target cost to explore the nature of search target representations. Journal of Experimental Psychology: Human Perception And Performance, 38(1), 113-122. https://doi.org/10.1037/a0025887

Stroud, M. J., Menneer, T., Cave, K. R., Donnelly, N., \& Rayner, K. (2011). Search for multiple targets of different colours: Misguided eye movements reveal a reduction of colour selectivity. Applied Cognitive Psychology, 25(6), 971-982. https://doi.org/10.1002/acp.1790

Treisman, A. M., \& Gelade, G. (1980). A feature-integration theory of attention. Cognitive Psychology, 12(1), 97-136.

Webster, M. A., \& Kay, P. (2012). Color categories and color appearance. Cognition, 122(3), 375-392.
Williams, D. E., \& Reingold, E. M. (2001). Preattentive guidance of eye movements during triple conjunction search tasks: The effects of feature discriminability and saccadic amplitude. Psychonomic Bulletin \& Review, 8(3), 476-488.

Williams, L. G. (1967). The effects of target specification on objects fixated during visual search. Acta Psychologica, 27, 355-360.

Wolfe, J. M. (2012). Saved by a log: How do humans perform hybrid visual and memory search? Psychol Sci, 23(7), 698-703. https://doi. org $/ 10.1177 / 0956797612443968$

Wolfe, J.M., Aiezenman, A., M., Boettcher, S., \& Cain, M. (2016). Hybrid foraging search: Searching for multiple instances of multiple types of target. Vision Research, 119, $50-59$.

Wolfe, J. M., Cave, K. R., \& Franzel, S. L. (1989). Guided search: An alternative to the feature integration model for visual search. Journal of Experimental Psychology: Human Perception and Performance, 15(3), 419-433.

Wolfe, J. M., \& Horowitz, T. S. (2004). What attributes guide the deployment of visual attention and how do they do it? Nature Reviews Neuroscience, 5(6), 495-501.

Wyszecki, G., \& Stiles, W. S. (1982). Color Science: Concepts and Methods, Quantitative Data andFormulae. 2nd. New York: Wiley. 